\title{
Article \\ Estimation of Structural Deformed Configuration for Bridges Using Multi-Response Measurement Data
}

\author{
Namju Byun ${ }^{1}\left(\mathbb{D}\right.$, Jeonghwa Lee ${ }^{2}\left(\mathbb{D}\right.$, Keesei Lee $^{3}$ and Young-Jong Kang ${ }^{1, * \mathbb{C}}$ \\ 1 School of Civil, Environmental and Architectural Engineering, Korea University, Seoul 02841, Korea; \\ skawn0702@naver.com \\ 2 Future and Fusion Laboratory of Architectural, Civil and Environmental Engineering, Korea University, \\ Seoul 02841, Korea; qevno@korea.ac.kr \\ 3 Department of Urban Infrastructure Research, Seoul Institute of Technology, Seoul 03909, Korea; \\ kslee@sit.re.kr \\ * Correspondence: yjkang@korea.ac.kr; Tel.: +82-2-3290-3317
}

check for updates

Citation: Byun, N.; Lee, J.; Lee, K.; Kang, Y.-J. Estimation of Structural Deformed Configuration for Bridges Using Multi-Response Measurement Data. Appl. Sci. 2021, 11, 4000. https://doi.org/10.3390/app11094000

Academic Editor:

Antonella D'Alessandro

Received: 19 March 2021

Accepted: 23 April 2021

Published: 28 April 2021

Publisher's Note: MDPI stays neutral with regard to jurisdictional claims in published maps and institutional affiliations.

Copyright: (c) 2021 by the authors. Licensee MDPI, Basel, Switzerland. This article is an open access article distributed under the terms and conditions of the Creative Commons Attribution (CC BY) license (https:// creativecommons.org/licenses/by/ $4.0 /)$.

\begin{abstract}
The structural deformed shape (SDS) is considered an important factor for evaluating structural conditions owing to its direct relationship with structural stiffness. Recently, an SDS estimation method based on displacement data from a limited number of data points was developed. Although the method showed good performance with a sufficient number of measured data points, application of the SDS estimation method for on-site structures has been quite limited because collecting sufficient displacement data measured from a Global Navigation Satellite System (GNSS) can be quite expensive. Thus, the development of an affordable SDS estimation method with a certain level of accuracy is essential for field application of the SDS estimation technique. This paper proposes an improved SDS estimation method using displacement data combined with additional slope and strain data that can improve the accuracy of the SDS estimation method and reduce the required number of GNSSs. The estimation algorithm was established based on shape superposition with various combined response data (displacement, slope, and strain) and the least-squares method. The proposed SDS estimation method was verified using a finite element method model. In the validation process, three important issues that may affect the estimation accuracy were analyzed: effect of shape function type, sensor placement method, and effectiveness of using multi-response data. Then, the improved SDS estimation method developed in this study was compared with existing SDS estimation methods from the literature. Consequently, it was found that the proposed method can reduce the number of displacement data required to estimate rational SDS by using additional slope and strain data. It is expected that cost-effective structural health monitoring (SHM) can be established using the proposed estimation method.
\end{abstract}

Keywords: SHM; estimation method; SDS; multi-response data

\section{Introduction}

As bridges across the world continue aging, structural health monitoring (SHM) systems are being established and operated in many developed countries [1-5]. In an SHM system, measurement data such as displacement, strain, acceleration, slope, temperature, and wind speed measured by each sensor are collected and managed. These measurement data can be used directly and indirectly to evaluate the conditions of a bridge. However, the use of measurement data remains ineffective because of the low development level of the utilization method for practical purposes. Therefore, a method for utilizing measurement data must be developed for the effective management of bridges.

For the SHM of infrastructures, various structural responses, such as displacement, slope, and strain, are generated by an external load. Among them, displacement can be considered as one of the representative responses and can be used to evaluate the integrity of a bridge. It has been used for integrity evaluation by comparing it with the 
deflection limit determined through various methods such as numerical analysis and load testing. Linear variable differential transformers (LVDTs) and laser Doppler vibrometers (LDVs) are frequently used to measure displacement, but they have limitations, such as the requirement of additional fixed reference points and sensitivity to the surrounding climate. To overcome these limitations, various studies have been conducted to indirectly estimate displacement using strain, slope, and acceleration data.

Acceleration in the response of the structure has been widely used in the study of dynamic displacement estimation, owing to its low price and convenience. Park et al. estimated the displacement through the double integration of acceleration [6]. To use the double integration method, the initial conditions of displacement and velocity should be considered. Displacement was estimated through iterative calculations, assuming that the initial displacement and average velocity were zero. On the other hand, Lee et al. proposed a method for estimating displacement without initial conditions in a way that minimizes the least-squares error between the assumed acceleration and measured acceleration [7]. The assumed acceleration is expressed as the central finite difference of displacement. The estimation method proposed in [7] was extended to increase the estimation accuracy and combine it with measurement data for other responses [8-10]. However, because the spatial parameter is not included in the acceleration response, there is a limitation in that only the displacement where the sensor is installed can be estimated.

Using the slope and strain data, which include spatial parameters, can overcome this limitation. Hou et al. estimated displacement based on the shape superposition method using slope data [11]. The shape function consisted of the power series and coefficient for the boundary condition, and the weight factors for each shape function were derived using the least-squares method. The estimation method of displacement using strain data was first proposed by [12] based on modal mapping. Modal mapping using the mode shape as a shape function is based on the superposition method. Shin et al. applied a theoretical mode shape composed of sine functions [13] for general use of the estimation method proposed by [12], and its effectiveness was verified by empirical experiments on various structures. Subsequently, a study using mode shape, derived by frequency analysis of an finite element method (FEM) model, was conducted to improve the accuracy of the estimated displacement [10]. It was mentioned that the mode shape derived from the FEM model was more useful than the theoretical model shape for estimating displacement. In addition, the modal mapping proposed by [12] was used as a basic method in various studies for estimating displacement [14-19].

To overcome the limitations of conventional displacement sensors, various studies have been conducted to indirectly estimate the displacement using other response measurement data. However, these studies only focused on the estimation of the displacement at a certain point. The structural deformed shape (SDS), including the global displacement of a structure, is more useful for evaluating structural conditions compared to the displacement at a certain point because it can be used as a displacement load to evaluate the internal force of the structure through inverse analysis. Recently, as Global Navigation Satellite Systems (GNSSs), which can compensate for the shortcomings of conventional displacement sensors, have been developed, studies on estimating SDS using displacement data have been conducted $[20,21]$.

Choi et al. estimated the SDS based on the shape superposition and least-squares method in the same way as in previous studies, but a structural shape was applied as a shape function instead of a mode shape [20,21]. Although the mode shape derived by frequency analysis is the dynamic response including mass and stiffness properties of the structure, mode shape does not represent deformed shape by loading. Alternatively, the structural shape based on the structural stiffness is derived by static analysis applying a unit load to each node of the FEM model. The effectiveness of the estimation algorithm was verified using an FEM model (beam, truss, and beam + truss). Although the method showed good performance with a sufficient number of measured data points, application of the SDS estimation method for on-site structures has been quite limited because collecting 
sufficient displacement data measured from a GNSS can be quite expensive. Thus, the development of an affordable SDS estimation method that can reduce the required number of GNSS is essential for the cost-effective field application of the SDS estimation technique.

Therefore, in this study, SDS estimation method using only displacement data developed by Choi et al. [20] was extended to use multi-response (displacement, slope, and strain) data in combination. The addition of slope and strain data can reduce the required number of GNSS and cost for estimating rational SDS, because slope and strain sensors are generally cheaper than GNSS. An estimation algorithm was developed based on shape superposition with various combined response data (displacement, slope, and strain) and the least-squares method, and the structural shape was used as a shape function. The proposed SDS estimation method was verified using an FEM model (beam, truss).

Additionally, three important issues that may affect the estimation accuracy were also reviewed during the verification process: first, comparison of shape function type (mode shape vs. structural shape); second, the placement method of the multi-response sensor; and third, the effectiveness of using multi-response data for SDS estimation. Comparisons of methodology between this study and the literature are listed in Table 1 . The effectiveness of the estimation algorithm was verified by comparing it with the existing method presented in [20]. As a result of the comparison, the proposed method can reduce the number of displacement data required to estimate rational SDS by using additional slope and strain data. It is expected that cost-effective structural health monitoring (SHM) can be established using the proposed estimation method.

Table 1. Comparison of methodologies between this study and previous studies.

\begin{tabular}{|c|c|c|c|c|c|}
\hline Classification & [7] & [12] & [11] & {$[20,21]$} & This Study \\
\hline $\begin{array}{l}\text { Used response } \\
\text { data }\end{array}$ & Acceleration & Strain & Slope & Displacement & $\begin{array}{l}\text { Displacement } \\
\text { Slope } \\
\text { Strain }\end{array}$ \\
\hline $\begin{array}{l}\text { Estimated } \\
\text { response }\end{array}$ & $\begin{array}{l}\text { Displacement at a } \\
\text { point where the } \\
\text { sensor is installed }\end{array}$ & $\begin{array}{l}\text { Displacement at a } \\
\text { certain point }\end{array}$ & $\begin{array}{l}\text { Displacement at a } \\
\text { certain point }\end{array}$ & $\begin{array}{c}\text { Structural } \\
\text { deformed shape }\end{array}$ & $\begin{array}{c}\text { Structural } \\
\text { deformed shape }\end{array}$ \\
\hline Estimation method & Least-square & $\begin{array}{c}\text { Shape } \\
\text { Superposition } \\
\text { /Least-square }\end{array}$ & $\begin{array}{c}\text { Shape } \\
\text { Superposition } \\
\text { /Least-square }\end{array}$ & $\begin{array}{c}\text { Shape } \\
\text { Superposition } \\
\text { /Least-square }\end{array}$ & $\begin{array}{c}\text { Shape } \\
\text { Superposition } \\
\text { /Least-square }\end{array}$ \\
\hline Shape function & - & Mode shape & Power series & Structural shape & Structural shape \\
\hline $\begin{array}{l}\text { Sensor placement } \\
\text { method }\end{array}$ & - & - & - & - & $\begin{array}{l}\text { EI-DPR-distance } \\
\text { method }\end{array}$ \\
\hline
\end{tabular}

\section{Estimation Algorithm}

A modal approach that does not include integration and differentiation processes is mainly used to estimate SDS. The modal approach, based on the shape superposition method, is a theory that has been used in various fields for the mechanical analysis of structures, and [12] first applied it to the estimation of SDS. Choi et al. defined the relationship between displacement and strain using a modal approach and developed an algorithm that can estimate displacement using strain data [20]. In addition, an estimation algorithm based on the shape-superposition method using displacement data was developed. In this study, the estimation algorithm proposed by [20] was extended based on [12] to additionally utilize slope and strain data.

In a linear problem, each response of the structure can be expressed as the product of the shape function $\Phi$ and weight factor $\alpha$ based on the shape superposition, as shown in Equations (1)-(3). The weight factors for displacement $u$, slope $\theta$, and strain $\epsilon$ are the 
same because the slope and strain are the first and second derivatives of displacement, respectively. This is the basic idea of an estimation algorithm that uses multi-response data.

$$
\begin{aligned}
& \{\mathrm{u}\}=\left[\Phi_{u}\right]\{\alpha\} \\
& \{\theta\}=\left[\Phi_{\theta}\right]\{\alpha\} \\
& \{\epsilon\}=\left[\Phi_{\epsilon}\right]\{\alpha\} .
\end{aligned}
$$

The composition system and analysis process of the estimation algorithm developed in this study are presented in Figure 1. The estimation process of the deformed shape consists of three steps: structure shape function composition (step 1), measurement of data (step 2), and deformed shape estimation (step 3). In step 1, an arbitrary superposed shape matrix (ADS) is constructed by using a shape function matrix $\Phi$ that consists of a structural shape or mode shape and weight factor. In step 2, a measurement data matrix MD is formed by combining the measurement data of displacement, slope, and strain. In step 3, the error function $\mathrm{E}$ is constructed by combining ADS and MD, and the weight factor that can minimize E is calculated using the least-squares method. Finally, the deformed shape can be estimated by applying the weight factor calculated in Equation (1).

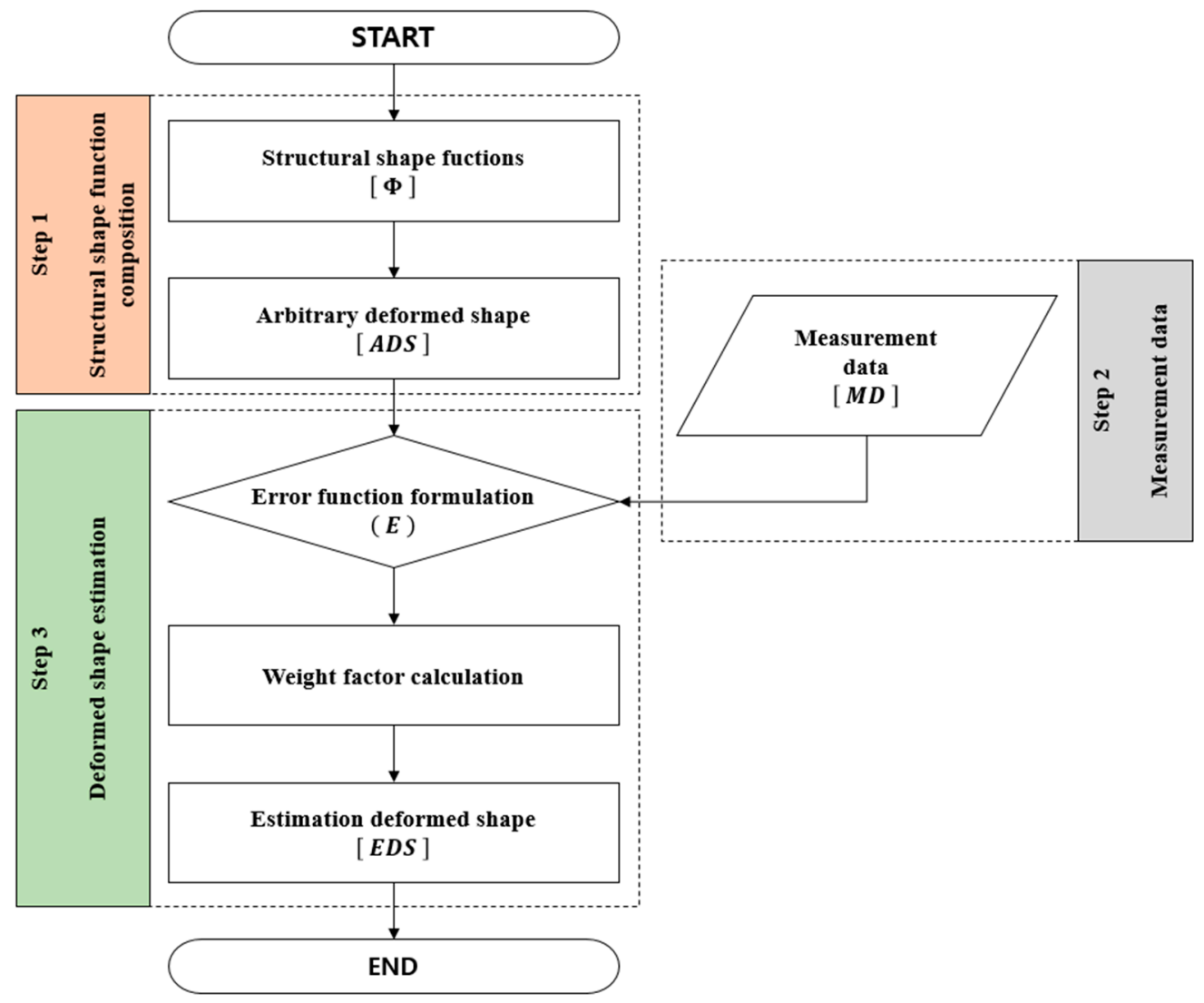

Figure 1. Analysis procedure of estimation algorithm for deformed shape.

\subsection{Step 1: Structural Shape Function Composition}

In this step, a system model for the target structure is constructed to derive the shape function. The mode shape function (MSF) derived by frequency analysis and a structural shape function (SSF) derived by static analysis applying a unit load on each node of the system model can be used as a shape function. Due to the fact that most structures are designed to operate within a linear range considering usability, the SSF is derived from a linear system model. The $i^{\text {th }}$ shape functions $\Phi_{i, u}, \Phi_{i, \theta}$, and $\Phi_{i, \epsilon}$ for displacement, slope, and strain are represented by Equations (4)-(6), respectively. Finally, the $i^{\text {th }}$ structural 
shape function $\Phi_{i}$ is derived by combining the shape function for each response, as shown in Equation (7).

$$
\begin{aligned}
& \Phi_{i, u^{T}}{ }^{T}=\left[\begin{array}{llllll}
u_{i 1} & u_{i 2} & u_{i 3} & u_{i 4} & \cdots & u_{i N_{d o f, u}}
\end{array}\right] \\
& \Phi_{i, \theta}{ }^{T}=\left[\begin{array}{llllll}
\theta_{i 1} & \theta_{i 2} & \theta_{i 3} & \theta_{i 4} & \cdots & \theta_{i N_{d o f, \theta}}
\end{array}\right] \\
& \Phi_{i, \epsilon}{ }^{T}=\left[\begin{array}{llll}
\epsilon_{i 1} & \epsilon_{i 2} \epsilon_{i 3} \epsilon_{i 4} & \cdots & \epsilon_{i N_{d o f, \epsilon}}
\end{array}\right] \\
& \Phi_{i}=\left[\begin{array}{c}
\Phi_{i, u} \\
\Phi_{i, \theta} \\
\Phi_{i, \epsilon}
\end{array}\right]\left(N_{\text {dof }} \times 1\right)
\end{aligned}
$$

where $N_{d o f}=N_{d o f, u}+N_{d o f, \theta}+N_{d o f, \epsilon}$ is the number of degrees of freedom; $N_{d o f, u}$, $N_{d o f, \theta}$, and $N_{d o f, \epsilon}$ are the numbers of degrees of freedom for each response, respectively; and $u_{i N_{d o f, u^{\prime}}} \theta_{i N_{d o f, \theta},}$ and $\epsilon_{i N_{d o f, \varepsilon}}$ are the $i^{t h}$ displacement, slope, and strain at each degree of freedom, respectively.

An arbitrarily deformed $\widetilde{A D S}$, as shown in Equation (8), is defined as a superposed shape function multiplied by the weight factor for each shape function. $N_{s f}$ is the number of shape functions considered for the deformed shape estimation. Equation (8) can be transformed into the matrix form shown in Equation (9).

$$
\begin{gathered}
\widetilde{A D S}=\alpha_{1} \Phi_{1}+\alpha_{2} \Phi_{2}+\alpha_{3} \Phi_{3}+\cdots+\alpha_{N_{s f}} \Phi_{N_{s f}}=\sum_{i=1}^{N_{s f}} \alpha_{i} \Phi_{i} \\
\widetilde{A D S}=\left[\begin{array}{c}
\alpha_{1} \\
\alpha_{2} \\
\alpha_{3} \\
\vdots \\
\alpha_{N_{s f}}
\end{array}\right]\left(\Phi_{3} \cdots \Phi_{N_{s f}} \times 1\right) .
\end{gathered}
$$

\subsection{Step 2: Measurement Data}

In this step, the measured data matrices for each response $M D_{\omega}, M D_{\varnothing}$, and $M D_{\varepsilon}$, as shown in Equations (10)-(12), are constructed by using on-site measured displacement data $\omega$, slope data $\varnothing$, and strain data $\varepsilon$, respectively. Owing to the limitation of the measured point number, each response data can be obtained only at finite points. $N_{m d, \omega}, N_{m d, \varnothing}$, and $N_{m d, \varepsilon}$ represent the number of measured data for each response, respectively. The total measured data matrix $M D$, as shown in Equation (13), is an $N_{m d} \times 1$ matrix that can be derived by combining the measured data for each response. The total number of measured data $N_{m d}$ is the sum of $N_{m d, \omega}, N_{m d, \varnothing}$, and $N_{m d, \varepsilon}$.

$$
\begin{aligned}
& M D_{\omega}{ }^{T}=\left[\omega_{1} \omega_{2} \omega_{3} \omega_{4} \cdots \omega_{N_{m d, \omega}}\right] \\
& M D_{\varnothing}{ }^{T}=\left[\varnothing_{1} \varnothing_{2} \varnothing_{3} \varnothing_{4} \cdots \omega_{N_{m d, \varnothing}}\right] \\
& M D_{\varepsilon}^{T}=\left[\begin{array}{llllll}
\varepsilon_{1} & \varepsilon_{2} & \varepsilon_{3} & \varepsilon_{4} & \cdots & \omega_{N_{m d, \varepsilon}}
\end{array}\right] \\
& M D=\left\{\begin{array}{c}
M D_{\omega} \\
M D_{\varnothing} \\
M D_{\mathcal{\varepsilon}}
\end{array}\right\}\left(N_{m d} \times 1\right) .
\end{aligned}
$$

\subsection{Step 3: Deformed Shape Estimation}

The weight factor required to estimate SDS can be calculated using $M D$ and $\widetilde{A D S}$. Owing to the different MD matrix sizes of $M D$ in Equation (13) and the $\overline{A D S}$ in Equation 
(9), it is necessary to adjust the matrix size of the $\widetilde{A D S}$ to construct an error function. To adjust the matrix size, $\widehat{A D S}$ is converted to $A D S$ by removing the row, excluding the data measurement point for each response. The least-squares method is adopted to calculate the weight factor that can minimize the error between $A D S$ and $M D$, and the calculation process is shown in Equations (14)-(16). The error function $E$ is constructed as the sum of the square errors between $A D S$ and $M D$ in Equation (14). To minimize $E$, the partial differential equation for the weight factor should be zero, as shown in Equation (15). Then, Equation (16) can be derived by substituting Equation (8) into Equation (15) and transformed into a matrix form as shown in Equation (17).

$$
\begin{gathered}
E=\sum_{j=1}^{N_{m d}}\left(M D_{j}-A D S_{j}\right)^{2} \\
\frac{\partial E}{\partial \alpha_{k}}=2 \sum_{j=1}^{N_{m d}}\left[\left(M D_{j}-A D S_{j}\right)\left(\frac{\partial M D_{j}}{\partial \alpha_{k}}-\frac{\partial A D S_{j}}{\partial \alpha_{k}}\right)\right]=0 \text { where } k=1,2, \cdots, N_{s f} \\
\frac{\partial E}{\partial \alpha_{k}}=\sum_{j=1}^{N_{m d}}\left[\left(\sum_{i=1}^{N_{s f}} \alpha_{i} \Phi_{i j}\right)\left(\Phi_{k j}\right)\right]=\sum_{j=1}^{N_{m d}}\left[\left(M D_{j}\right)\left(\Phi_{k j}\right)\right] \text { where } k=1,2, \cdots, N_{s f} \\
{[\Phi]_{N_{m d} \times N_{s f}}^{T}[\Phi]_{N_{m d} \times N_{s f}}\{\alpha\}_{N_{s f} \times 1}=[\Phi]_{N_{m d} \times N_{s f}}^{T}\{M D\}_{N_{m d} \times 1} .}
\end{gathered}
$$

The weight factor that minimizes the square error between $A D S$ and $M D$ can be calculated from Equation (17). Foss and Haugse used MSF and proposed the calculation of the weight factor using the inverse matrix of the $[\Phi]^{T}[\Phi]$ term [12]. However, if the number of shape functions is larger than the number of measured data, then the inverse matrix cannot be derived because $[\Phi]^{T}[\Phi]$ becomes a rank-deficient matrix. In general, the amount of data that can be measured on a structure is limited owing to the cost. In the case of using MSF, the inverse matrix can be adopted because a small number of shape functions are used.

When using the SSF derived by applying a unit load to each node of the FEM model, a different method is required to calculate the weight factor. To solve this problem, in this case, the singular value decomposition (SVD) method [22] was adopted to calculate the weight factor. The rank-deficient linear algebra problem can be solved reliably by using the SVD method. Finally, the estimated deformed shape (EDS) can be obtained by substituting the calculated weight factor into the displacement part of Equation (9).

\section{Validation Issues and FEM Model}

\subsection{Validation Process and Issues}

The FEM model (beam and truss) was used to verify the developed algorithm, and the validation flow is shown in Figure 2. A real deformed shape (RDS) is assumed by applying various static load conditions to the FEM model, and the MD matrix is constructed from the displacement, slope, and strain values at a specific location in the RDS. Due to the fact that the data measured from the on-site structure include measurement errors, differences from the FEM model may occur. However, because the algorithm proposed in this paper is developed by extending the existing algorithm already verified in various studies, and the objective of this study was to validate the algorithm, measurement errors that occur in the field were not considered here.

As multi-response data are used in the developed algorithm, the following three issues are analyzed and reviewed to derive appropriate estimation results: shape function type, sensor placement method, and EDS by multi-response data. First, an appropriate shape function type should be selected to properly estimate the deformed shape. The MSF derived from the frequency analysis and SSF derived from the static analysis can be used as a shape function. An appropriate shape function is selected by comparing the estimation 
results of MSF and SSF through the data at an arbitrarily selected position. Second, when multi-response measurement data are used for shape estimation, interference between the data may affect the estimation results. Various methods for optimal sensor placement (OSP) have been proposed, but most of them consider only the data of one response. To solve this issue, a new sensor placement method is proposed in this study, and the estimation results of various sensor placement methods were compared. Third, the effectiveness of the algorithm developed in this study was verified by comparing the estimation error through multi-response data and that using only displacement data.

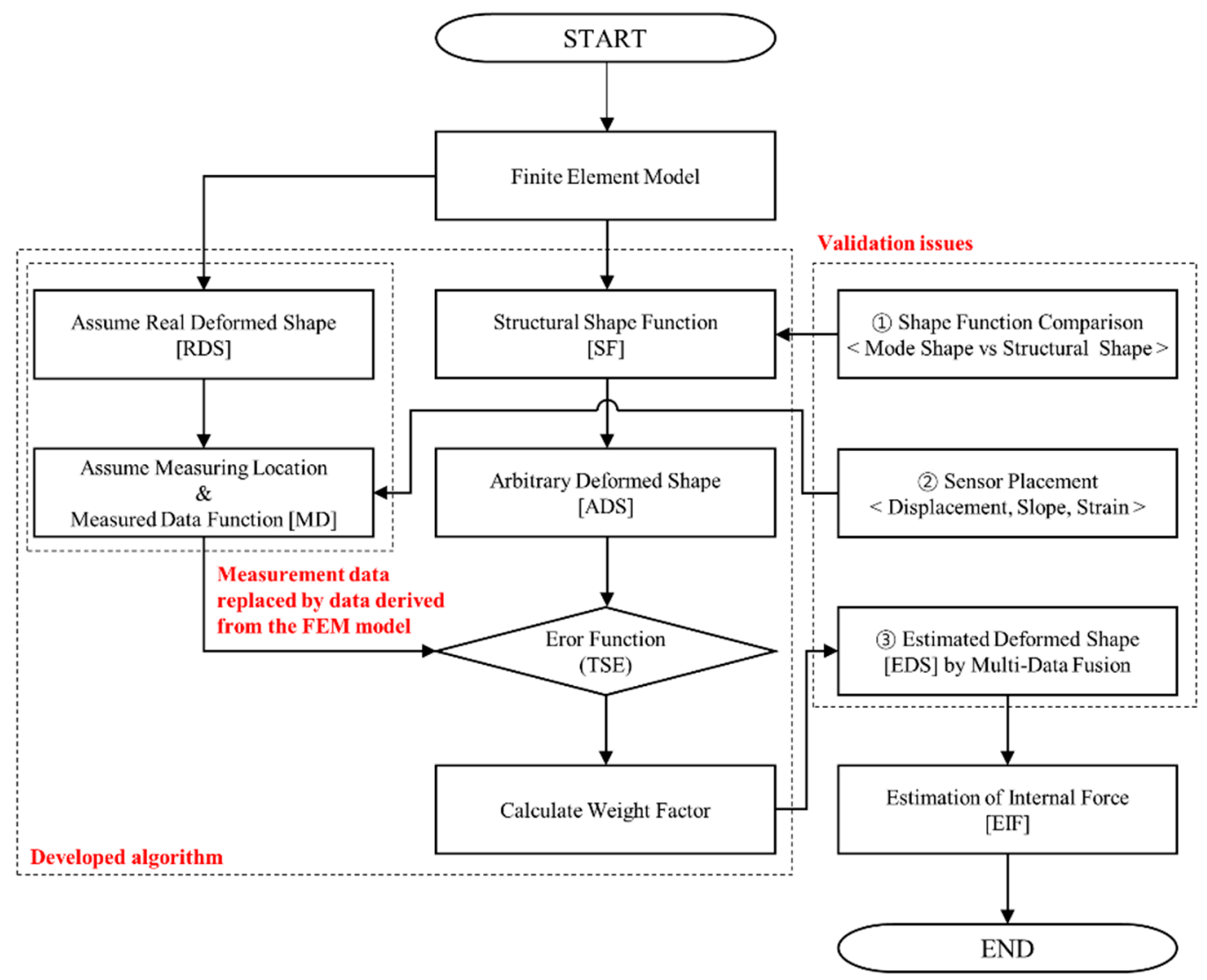

Figure 2. Validation procedure through finite element method (FEM) model considering three issues.

The mean absolute percent error (MAPE) and mutual correspondence criterion (MCC) are usually used as indices to indicate the error of the estimated deformed shape. However, MAPE is sensitive to small values and insensitive to large values, whereas MCC is sensitive to large values and insensitive to small values. Therefore, in this study, normalized MAPE (NMAPE) was used as the error index to analyze the absolute estimation error between RDS and EDS.

$$
\operatorname{NMAPE}(\%)=\frac{100}{N_{d o f, u}} \sum_{i=1}^{N_{d o f, u}} \frac{\left|R D S_{i}-E D S_{i}\right|}{|R D S|_{\max }} .
$$

\subsection{Numerical Model for Verification}

To verify the developed algorithm, a beam model with a relatively simple geometric shape and a truss model with a complex geometric shape were selected as the numerical analysis models. The beam model in Figure 3, a four-span structure with a total length of $75 \mathrm{~m}$, is composed of 61 nodes and 60 elements. The truss model in Figure 4, a two-span structure with a total length of $120 \mathrm{~m}$, consists of 24 nodes and 55 elements. In addition, in order to analyze the estimation results of various deformed shapes that can occur according to the load conditions, four load conditions are considered as RDS1-4 in each numerical analysis model, as shown in Figures 5 and 6. Each load condition is configured by 
combining concentrated loads $P_{1}$ and $P_{2}$ of different magnitude and uniformly distributed loads. The magnitude of the uniformly distributed load is determined by dividing $P_{1}$ and $P_{2}$ by the number of nodes where the uniformly distributed load is applied. The number in parentheses indicates the number of the node to which the load is applied.

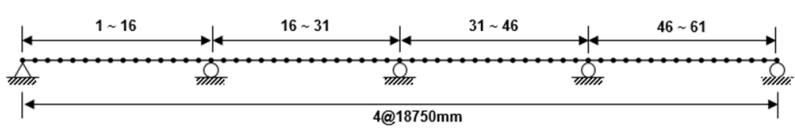

(a)

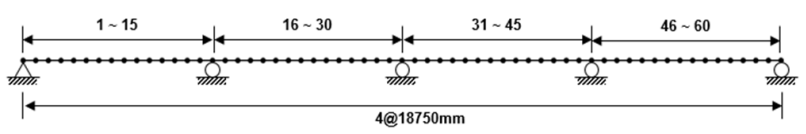

(b)

Figure 3. FEM model for validation (beam): (a) node number, (b) element number.

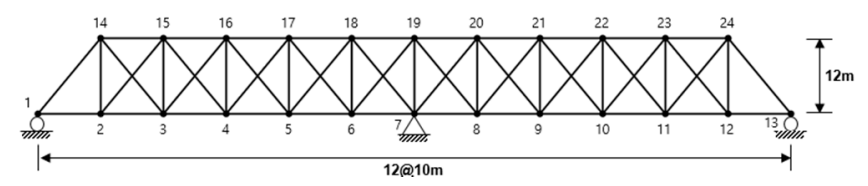

(a)

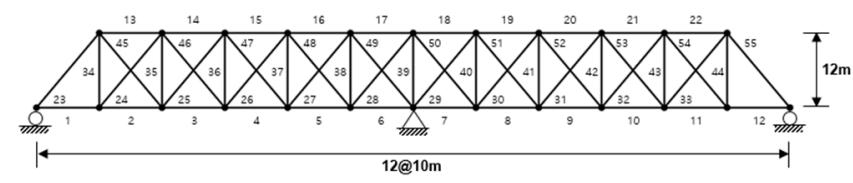

(b)

Figure 4. FEM model for validation (truss): (a) node number, (b) element number.

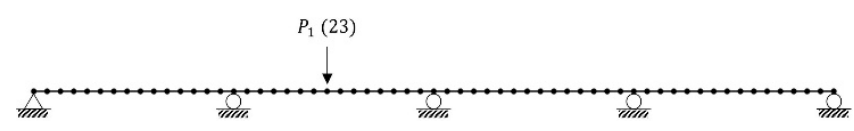

(a)

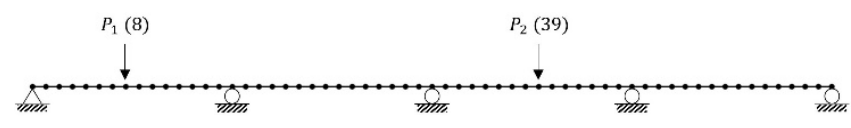

(b)

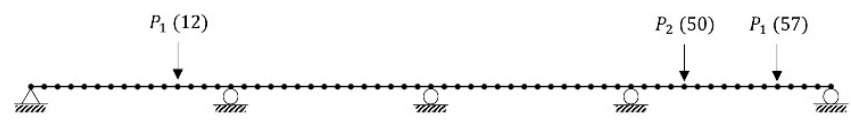

(c)

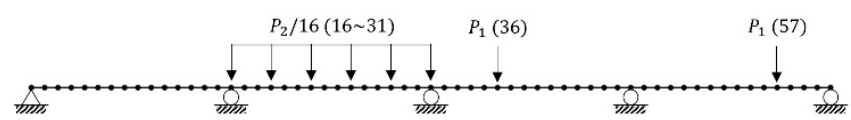

(d)

Figure 5. Load conditions for real deformed shape assumed by FEM model (beam): (a) RDS1, (b) RDS2, (c) RDS3, (d) RDS4. 


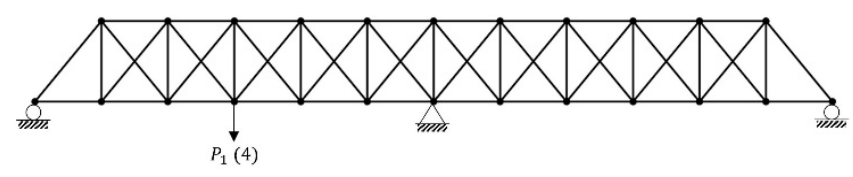

(a)

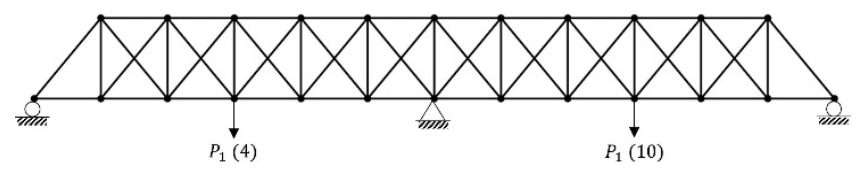

(b)

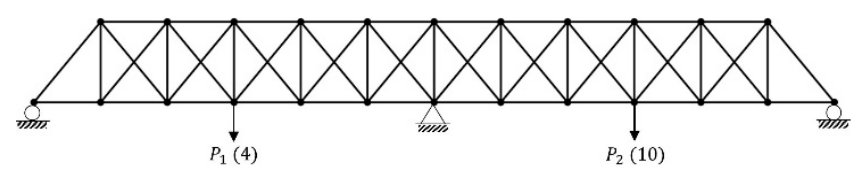

(c)

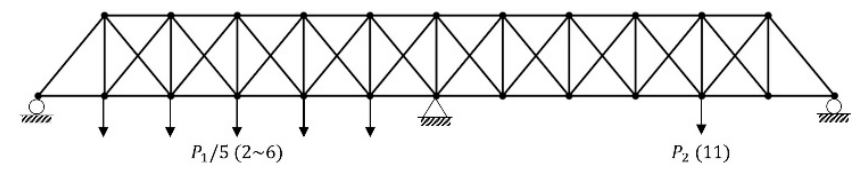

(d)

Figure 6. Load conditions for real deformed shape assumed by FEM model (truss): (a) RDS1, (b) RDS2, (c) RDS3, (d) RDS4.

\section{Validation of Results}

\subsection{Effect of Shape Function Type}

As previously mentioned, the shape function type can affect the accuracy of the estimation results. An MSF derived by frequency analysis and SSF derived by static analysis can be used as a shape function. In the case of the MSF used by [12], because global deformation is included in one mode shape, an appropriate estimation accuracy can be obtained through several low-order mode shapes. However, because the mode shape is not deformed owing to the load, the estimation result is sensitive to the number of used MSFs. In addition, the minimum number of sensors should be the same as the number of MSFs to derive the inverse matrix. Therefore, the lowest value of the errors estimated by using all the number of MSFs smaller than the number of sensors is considered as the estimation result of the deformed shape.

In the case of the SSF used in [20], because only local deformation near the point where the unit load is applied is included in one structural shape, all SSFs should be used to secure the estimation accuracy for various load conditions. In addition, [20] noted that the estimation accuracy converges to a certain value as the number of SSFs used increases. Therefore, the estimation results obtained using the SSF are represented in terms of an error value estimated by using all shape functions to ensure uniform accuracy.

\subsubsection{Beam Model}

Figures 7 and 8 show examples of the MSF and SSF for the four-span beam model. The total number of used MSFs is six, and the total number of used SSFs is 56. The SSF is derived by applying a unit load on all nodes except the boundary points. The response data of displacement, slope, and strain were used individually to analyze the effect of the shape function type on the estimation result for each response. The number of response data is 1-6 for the displacement and slope, and 1-5 for the slope. In addition, the sensor location, as shown in Table 2, is arbitrarily selected as the point at which the largest response can occur. 


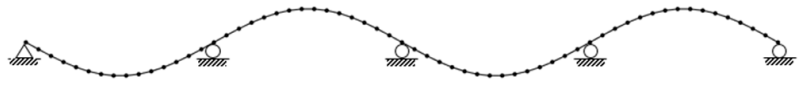

(a)

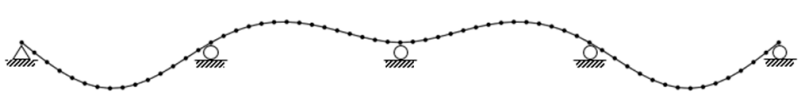

(b)

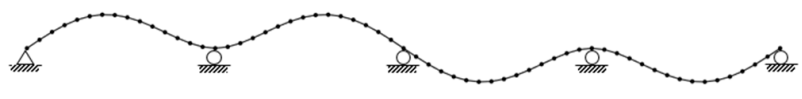

(c)

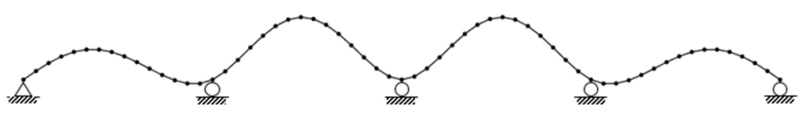

(d)

Figure 7. Examples of mode shape function (MSF) derived by frequency analysis (beam): (a) MSF1, (b) MSF2, (c) MSF3, (d) MSF4.

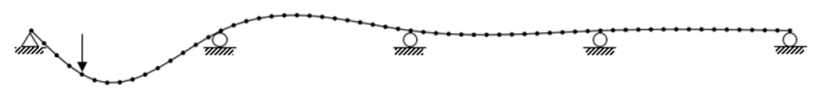

(a)

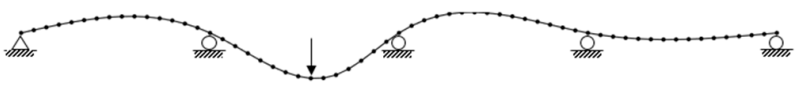

(b)

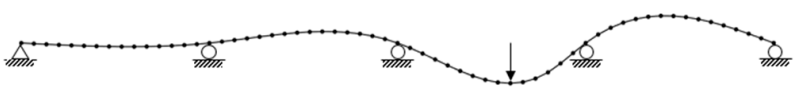

(c)

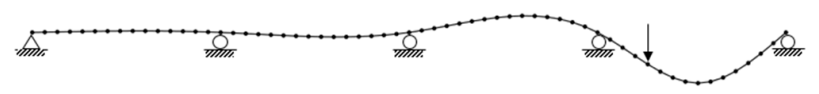

(d)

Figure 8. Examples of structural shape function (SSF) derived by static analysis (beam): (a) SSF4, (b) SSF22, (c) SSF37, (d) SSF46.

Table 2. Sensor location in the FEM model used for estimation (beam).

\begin{tabular}{ccccccc}
\hline \multirow{2}{*}{ Measured Data } & \multicolumn{7}{c}{ Number of Measured Data $\left(N_{\boldsymbol{m} \boldsymbol{d}}\right)$} \\
\cline { 2 - 7 } & $\mathbf{1}$ & $\mathbf{2}$ & $\mathbf{3}$ & $\mathbf{4}$ & $\mathbf{5}$ & $\mathbf{6}$ \\
\hline Displacement (Node) & 8 & 54 & 23 & 39 & 11 & 51 \\
Slope (Node) & 1 & 61 & 31 & 16 & 46 & - \\
Strain (Element) & 8 & 54 & 23 & 38 & 16 & 45 \\
\hline
\end{tabular}

The estimation error for EDS of RDS1-4 using each response data is shown in Figure 9. However, because the trend of the results according to MSF and SSF does not appear clearly, the number of sensors required to estimate the deformed shape within a $5 \%$ error is additionally shown in Figure 10. In Figure 10, the transparent bar indicates that more than six sensors are required to secure an NMAPE within 5\%. In the case of using displacement 
data, there is a difference in the accuracy trend according to the number of sensors, but the overall results show that SSF is more useful for RDS1, RDS3, and RDS4. When slope data were used, the estimation results corresponding to the MSF were found to be more accurate for all RDSs except RDS3. In Figures $9 \mathrm{c}$ and 10c, which represent the estimation results obtained using strain data, the SSF is generally useful for all RDSs except RDS2. As a result of the deformed shape estimation according to the shape function type for the beam model, the specific shape function type is not always useful for estimation. However, when MSF is used, an additional process for calculating the optimal number of MSFs is required to ensure an appropriate estimation accuracy, because the high-order mode shapes are considerably different from the deformed shape that can be caused by the load. Therefore, the SSF is more useful for estimating the deformed shape of the beam model in general owing to its convenience of use.

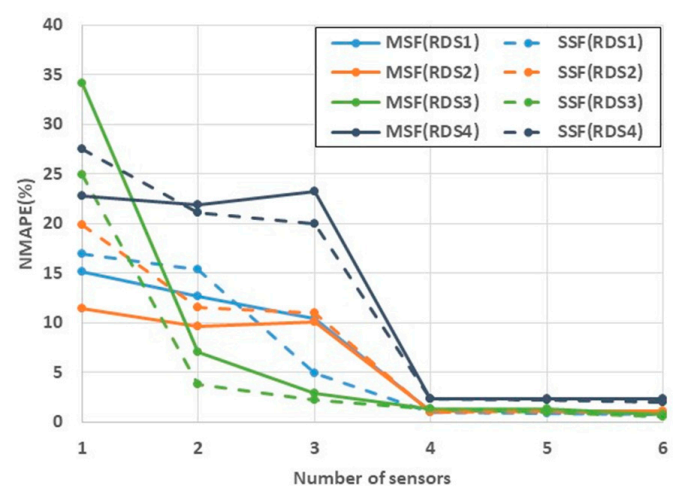

(a)

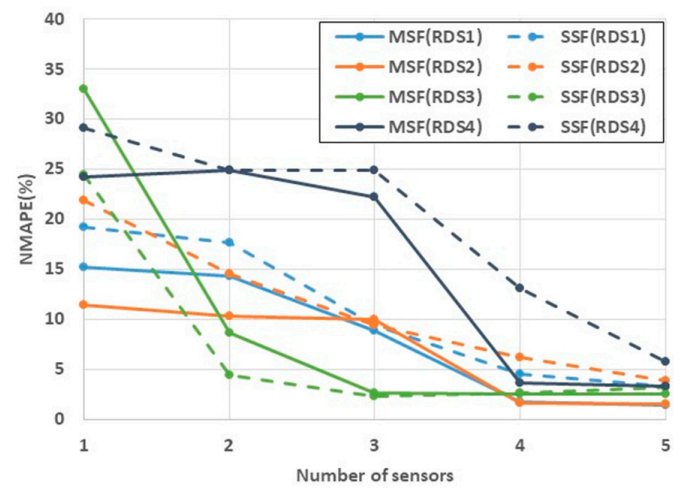

(b)

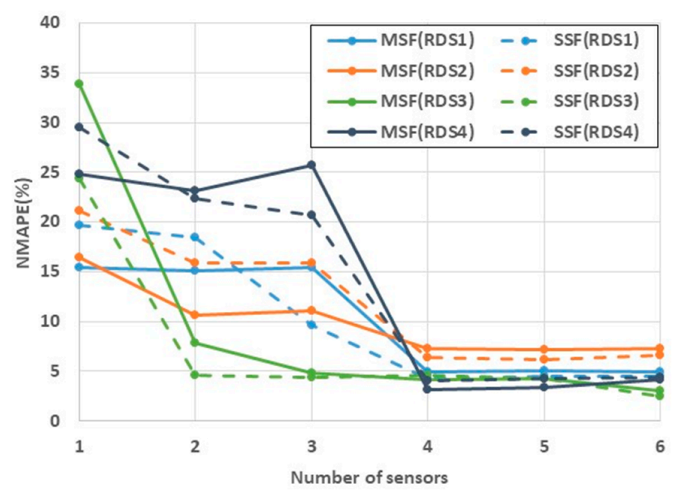

(c)

Figure 9. Normalized mean absolute percent error (NMAPE) by MSF and SSF according to the number of sensors: (a) displacement, (b) slope, (c) strain. 


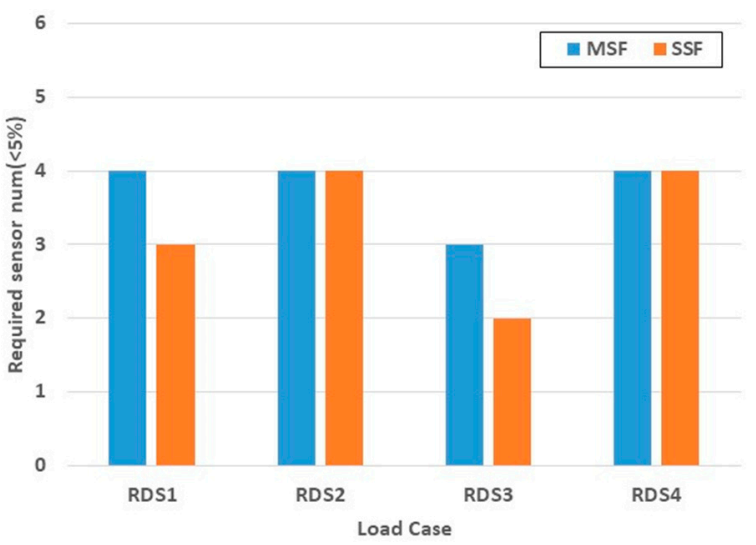

(a)

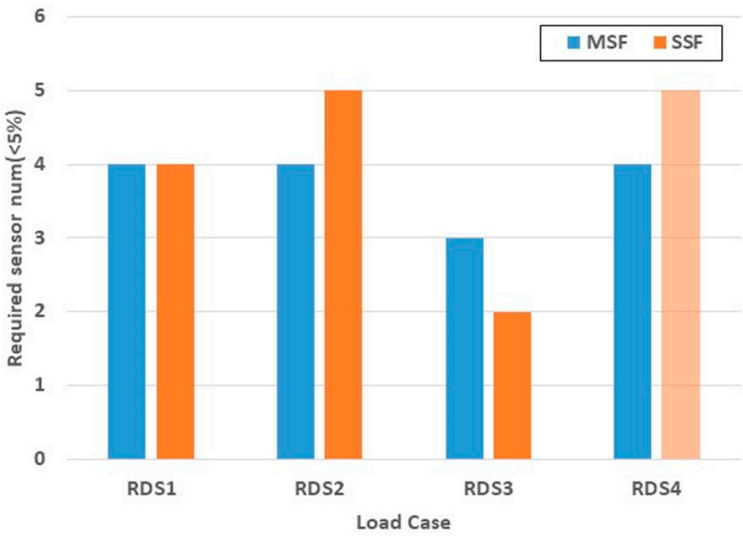

(b)

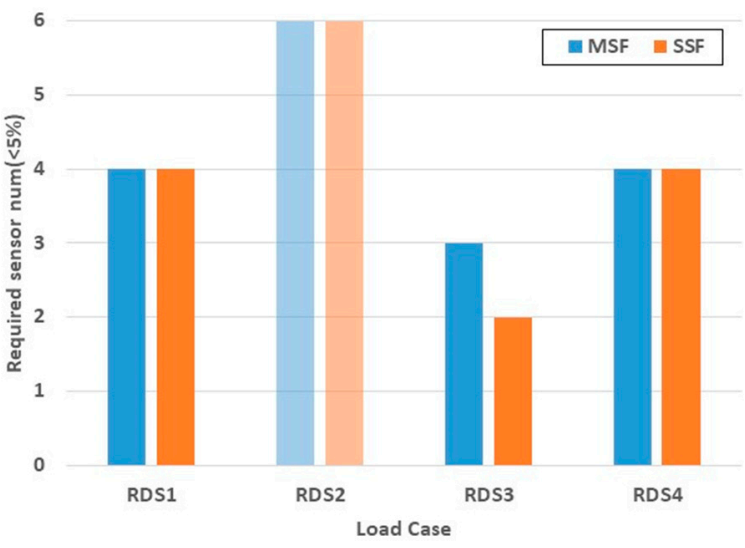

(c)

Figure 10. Required number of sensors (NMAPE < 5\%): (a) displacement; (b) slope; (c) strain.

\subsubsection{Truss Model}

Examples of MSF and SSF for the two-span truss model are shown in Figures 11 and 12, respectively. The total number of used MSFs is six, and the total number of used SSFs is 21. The SSF is derived by applying a unit load in the vertical direction to all nodes except the boundary points. In the same way as the beam model, the displacement, slope, and strain are used as response data. The number of data points is 1-6 for all responses. In addition, the sensor location, as shown in Table 3, is arbitrarily selected as the point at which the largest response can occur. 


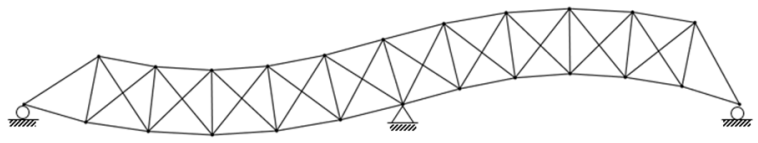

(a)

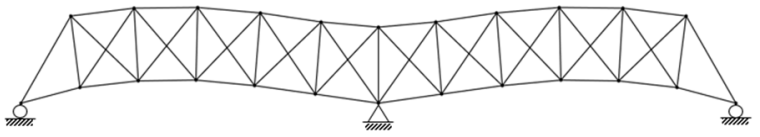

(b)

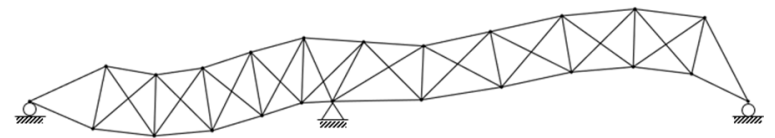

(c)

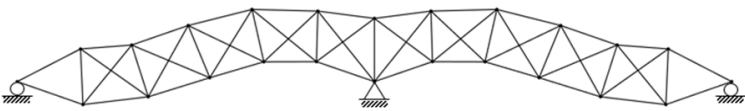

(d)

Figure 11. Examples of MSF derived by frequency analysis (truss): (a) MSF1, (b) MSF2, (c) MSF3, (d) MDF3.

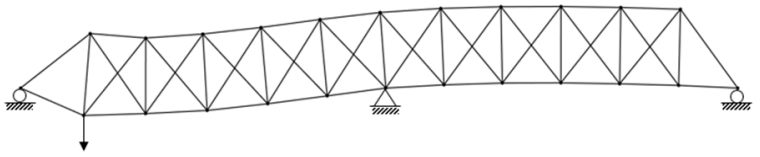

(a)

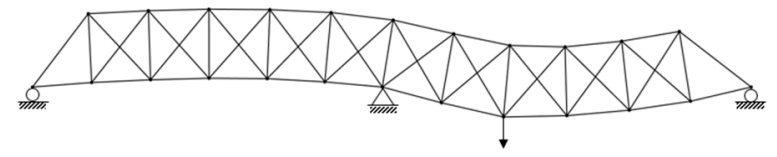

(b)

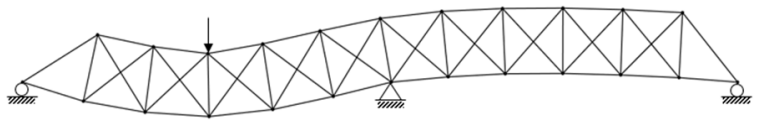

(c)

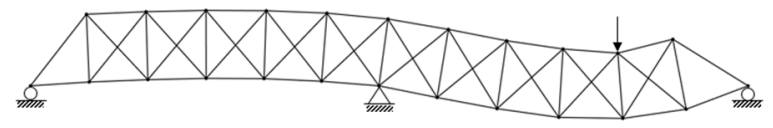

(d)

Figure 12. Examples of SSF derived by static analysis (truss): (a) SSF1, (b) SSF7, (c) SSF13, (d) SSF20.

For all RDSs, the NMAPE of the deformed shape estimated using the response data is shown in Figure 13, and the number of sensors required to secure NMAPE within 5\% is shown in Figure 14. In contrast to the beam model, for the truss model, which has a complex geometric shape, the overall estimation results obtained using the SSF are more accurate than those obtained using the MSF. If the geometric shape of the numerical analysis model is complex, then the deformed shape generated by the load cannot be sufficiently expressed 
by the combination of MSF. In addition, it takes considerable time to analyze and select the optimal number of MSFs for each estimation case. Therefore, the SSF is used to analyze the sensor placement method and the effectiveness of using multi-response data.

Table 3. Sensor location in the FEM model used for estimation (truss).

\begin{tabular}{ccccccc}
\hline Measured Data & \multicolumn{7}{c}{ Number of Measured Data $\left(\boldsymbol{N}_{\boldsymbol{m} \boldsymbol{d}}\right)$} \\
\cline { 2 - 7 } & $\mathbf{1}$ & $\mathbf{2}$ & $\mathbf{3}$ & $\mathbf{4}$ & $\mathbf{5}$ & $\mathbf{6}$ \\
\hline Displacement (Node) & 4 & 10 & 16 & 22 & 3 & 11 \\
Slope (Element) & 1 & 12 & 6 & 7 & 13 & 22 \\
Strain (Element) & 3 & 10 & 15 & 20 & 5 & 8 \\
\hline
\end{tabular}

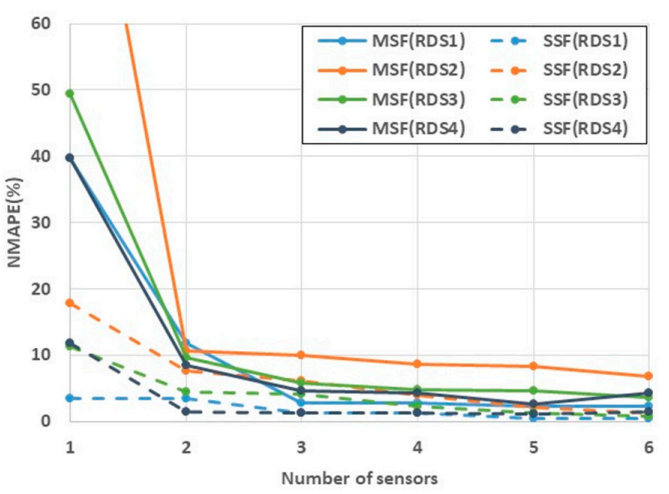

(a) Displacement

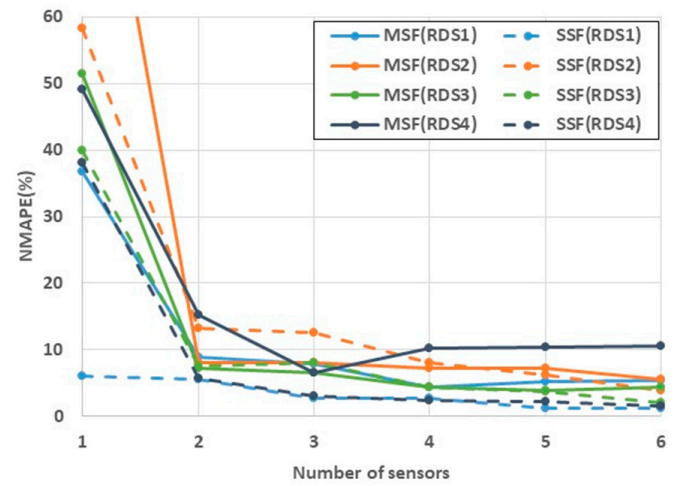

(b) Slope

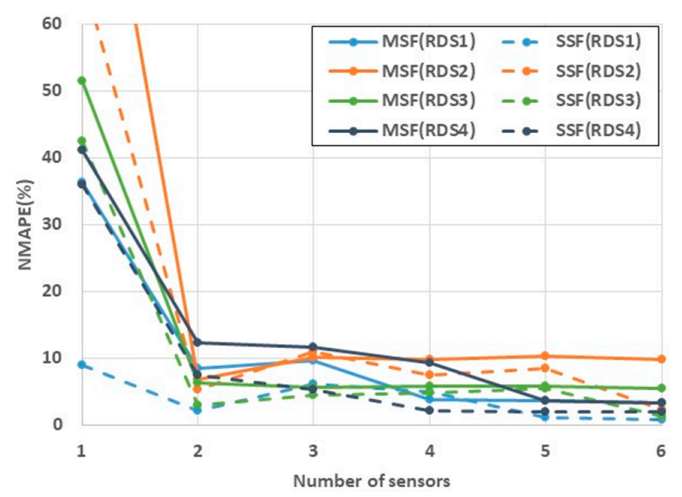

(c) Strain

Figure 13. NMAPE by MSF and SSF according to the number of sensors: (a) displacement, (b) slope, (c) strain. 


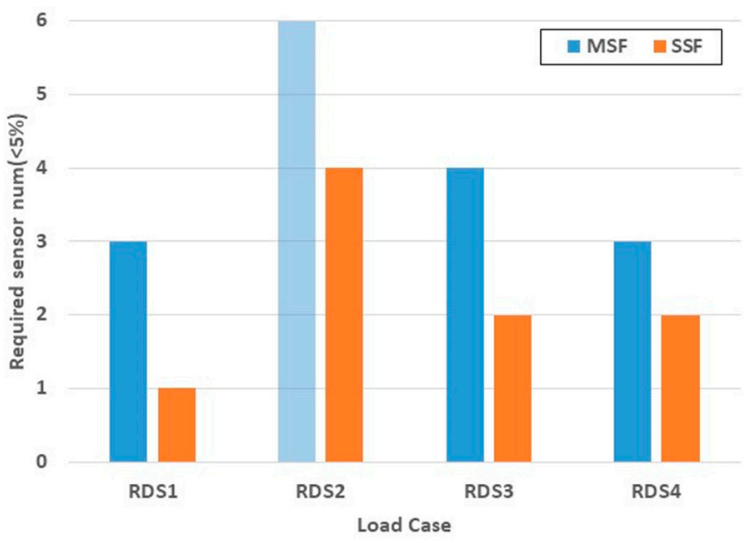

(a) Displacement

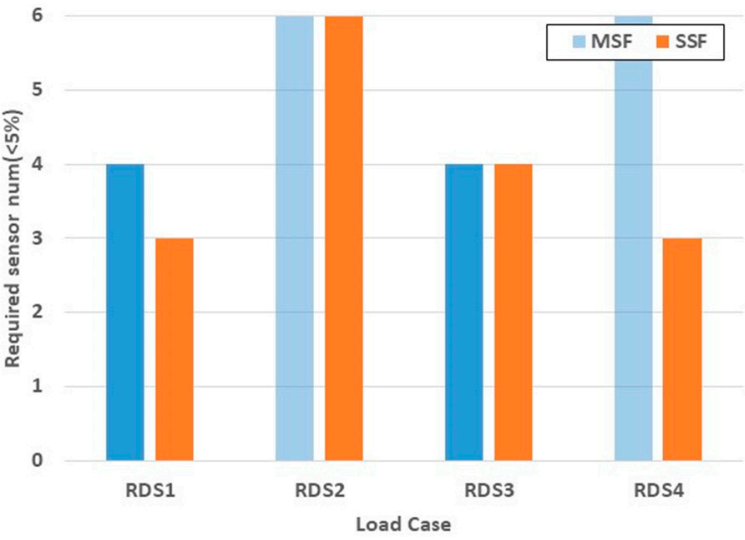

(b) Slope

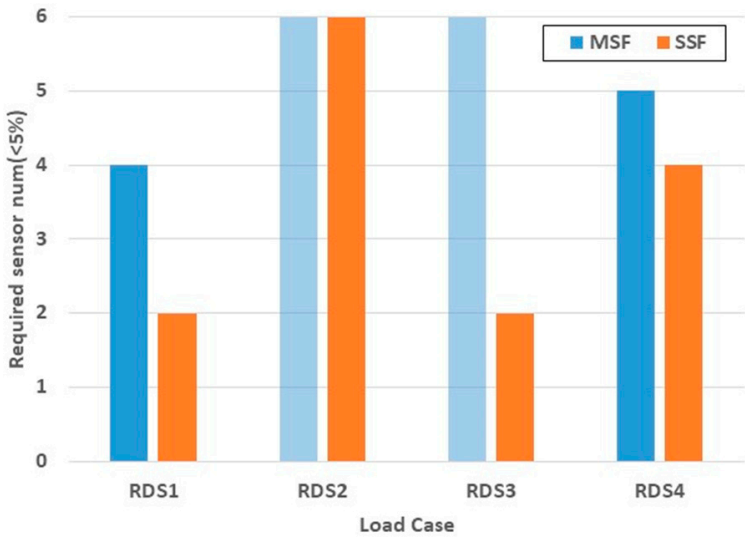

(c) Strain

Figure 14. Required number of sensors by MSF and SSF (NMAPE < 5\%): (a) displacement, (b) slope, (c) strain.

\subsection{Sensor Placement Method}

\subsubsection{Methods of Sensor Placement}

To ensure the accuracy of the deformed shape estimation, the response data should be measured at an appropriate location. Research on the OSP method must been conducted in various fields before the need for SHM can be recognized. Representatively, [23] applied six different OSP methods to estimate the deformed shape of a suspension bridge and compared the estimation results. The OSP methods used for comparison were effective independence (EI), EI-driving point residue (EI-DPR), kinetic energy method (KEM), variance 
method (VM), eigenvalue vector product (EVP), and nonoptimal driving point (NODP). As a result of comparisons, the estimation results obtained based on EI-DPR showed the best performance. The EI-DPR method has been used in various studies on sensor placement methods $[24,25]$. Therefore, in this study, a sensor placement method for deformed shape estimation using SSF and multi-response data was analyzed based on the EI method.

The EI sensor placement method [26] was developed to maximize both the spatial independence and signal strength of the shape function by maximizing the determinant of the associated Fisher information matrix. To maximize the determinant of the Fisher information matrix, the effective independence distribution (EID) $E_{D}$ is derived as a diagonal of Equation (19). The $i$ th component of $E_{D}$, described in Equation (20), indicates the fractional contribution to the shape function at the $i$ th sensor location. A sensor location noted as the lowest index of $E_{D}$ is eliminated from the candidate locations, and this procedure is repeated until the remaining number of candidate locations is the same as the determined number of sensors.

$$
\begin{gathered}
{[E]=[\Phi]\left([\Phi]^{T}[\Phi]\right)^{-1}[\Phi]^{T}} \\
E_{D_{i}}=\operatorname{diagonal}([E])_{i} .
\end{gathered}
$$

Papadopoulos and Garcia proposed a driving point residue (DPR) coefficient to overcome the limitations of the EI method, which allows the selection of sensor locations associated with low energy content [27]. The DPR coefficient, as described in Equation (21), is a weight factor that considers the modal contribution at each sensing location. The $j$ th modal frequency $\omega_{j}$ is applied to consider a higher weight factor for the higher-order mode. Then, $E_{D}$ from Equation (20) can be transformed into Equation (22) by multiplying it by the DPR coefficient. EI-DPR is a method that concentrates sensor positions in high-energycontent regions.

$$
\begin{gathered}
D P R_{i}=\sum_{j=1}^{N_{s f}} \frac{\Phi_{i j}^{2}}{\omega_{j}}(M S F) \\
E_{D_{i}}=\operatorname{diagonal}([E])_{i} \times D P R_{i} .
\end{gathered}
$$

Although the EI and EI-DPR methods have been verified by various researchers, there is a limitation, in that they cannot be applied to an SSF with $N_{s f}>N_{m d}$ because of the $\left([\Phi]^{T}[\Phi]\right)^{-1}$ term in Equation (19). In addition, when using various response data, the contribution of overlapping and interference affects the relationship between response data. Therefore, in this paper, the DPR coefficient for the SSF and distance coefficient $d$ are proposed as Equations (23) and (24) to estimate the deformed shape using the SSF. To derive the DPR coefficient for SSF, the maximum value of the absolute $j$ th shape function $\left|\Phi_{j}\right|_{\text {max }}$ is used instead of $\omega_{j}$. The distance coefficient $d_{i}$ is the minimum value among the distances from the $i$ th candidate location to each sensor location previously selected.

The sensor locations previously selected contain locations that have a constant certain response value, such as the boundary for displacement. $E_{D}$, proposed in this study, was calculated using Equation (24) by multiplying the distance coefficient using Equation (22). The sensor location, which has the highest value of $E_{D}$, was selected, and the distance coefficient was recalculated containing the sensor location recently selected. This procedure was repeated until the selected number of sensors for each response was the same as the planned number of sensors. Using the $E_{D}$ proposed in this study, the limitations of the EI and EI-DPR methods and problems that may occur when using multi-response data can be overcome.

$$
\begin{gathered}
D P R_{i}=\sum_{j=1}^{N_{s f}} \frac{\Phi_{i j}^{2}}{\left|\Phi_{j}\right|_{\max }}(L S F) \\
E_{D_{i}}=\text { diagonal }([E])_{i} \times D P R_{i} \times d_{i} .
\end{gathered}
$$




\subsubsection{Comparison of Results}

As previously mentioned, the estimation results obtained using the SSF are generally more accurate than those obtained using the MSF. Therefore, in this study, the EI-DPRdistance method that can be applied to SSFs with $N_{s f}>N_{m d}$ is proposed and compared with the EI and EI-DPR methods. Due to the fact that the EI and EI-DPR methods cannot be applied when all SSFs are used, the SSFs that included the maximum value for each response, which correspond to the number of sensors, were selected and applied.

A truss model with a relatively complex geometric shape was used for comparison of the sensor placement method, and slope only (case 1) and strain with one displacement (case 2) were considered for the sensor combination. In case 2, the position of the displacement sensor was fixed at the 22th node in Figure 4a. The positions of the sensors selected by each sensor placement method are shown in Tables 4 and 5, and the selection procedure of sensor locations for case 2 is shown in Figure 15. In Figure 15, the normalized $E_{D}$ was used to compare the $E_{D}$ value of each sensor placement method under the same conditions.

Table 4. Sensor location in the FEM model used for estimation (Case 1: slope only).

\begin{tabular}{ccccccc}
\hline \multirow{2}{*}{ Optimization Method } & \multicolumn{7}{c}{ Number of Slope Measured Data $\left(\boldsymbol{N}_{\boldsymbol{m d}}\right)$} \\
\cline { 2 - 7 } & $\mathbf{1}$ & $\mathbf{2}$ & $\mathbf{3}$ & $\mathbf{4}$ & $\mathbf{5}$ & $\mathbf{6}$ \\
\hline EI & 1 & 12 & 14 & 10 & 21 & 3 \\
EI-DPR & 1 & 12 & 13 & 11 & 22 & 2 \\
EI-DPR-distance & 1 & 12 & 17 & 7 & 18 & 6 \\
\hline
\end{tabular}

Table 5. Sensor location in the FEM model used for estimation (Case 2: strain with one displacement).

\begin{tabular}{ccccccc}
\hline \multirow{2}{*}{ Optimization Method } & \multicolumn{7}{c}{ Number of Strain Measured Data $\left(\boldsymbol{N}_{\boldsymbol{m} \boldsymbol{d}}\right)$} \\
\cline { 2 - 7 } & $\mathbf{1}$ & $\mathbf{2}$ & $\mathbf{3}$ & $\mathbf{4}$ & $\mathbf{5}$ & $\mathbf{6}$ \\
\hline EI & 46 & 36 & 32 & 3 & 42 & 10 \\
EI-DPR & 23 & 34 & 55 & 3 & 44 & 10 \\
EI-DPR-distance & 23 & 39 & 55 & 4 & 14 & 10 \\
\hline
\end{tabular}

In the EI and EI-DPR methods, the nodes with the smallest $E_{D}$ are removed one by one for each iteration until the number of remaining nodes is the same as the number of sensors. In addition, the influence of the previously selected displacement sensor is not considered in the EI and EI-DPR methods. By contrast, in the EI-DPR-distance method proposed in this study, the node with the highest $E_{D}$ is selected as the sensor location for each iteration, and the location of the next sensor is determined by recalculating the distance coefficient considering the recently selected sensor. Therefore, the EI-DPR-distance method is a more effective sensor placement method when using multi-response data because the influence of different response sensors previously selected can be considered.

For cases 1 and 2, the shape estimation results according to the number of sensors are shown in Figures 16 and 17, respectively. Case 2, which additionally used one displacement data, generally showed more accurate results for all RDSs except RDS1. In case 1, the estimation error corresponding to the EI-DPR-distance method was the lowest for RDS4, and the estimation errors corresponding to all sensor placement methods were similar for RDS1-3. In case 2, the estimation errors corresponding to EI-DPR and EI-DPR-distance for all RDS were similar, but the estimation errors for EI depended on the RDS and number of sensors. In particular, for RDS2 and RDS3, the estimation errors increased despite the increase in the number of sensors. This phenomenon occurred for RDS4 in Case 1, even when EI-DPR was used. 


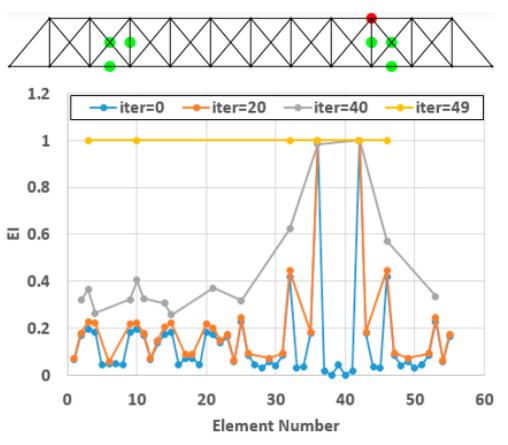

(a)

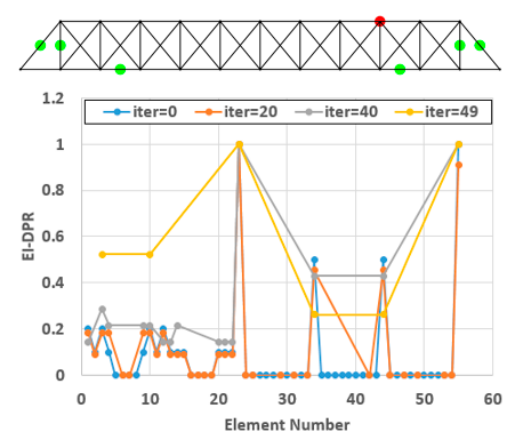

(b)
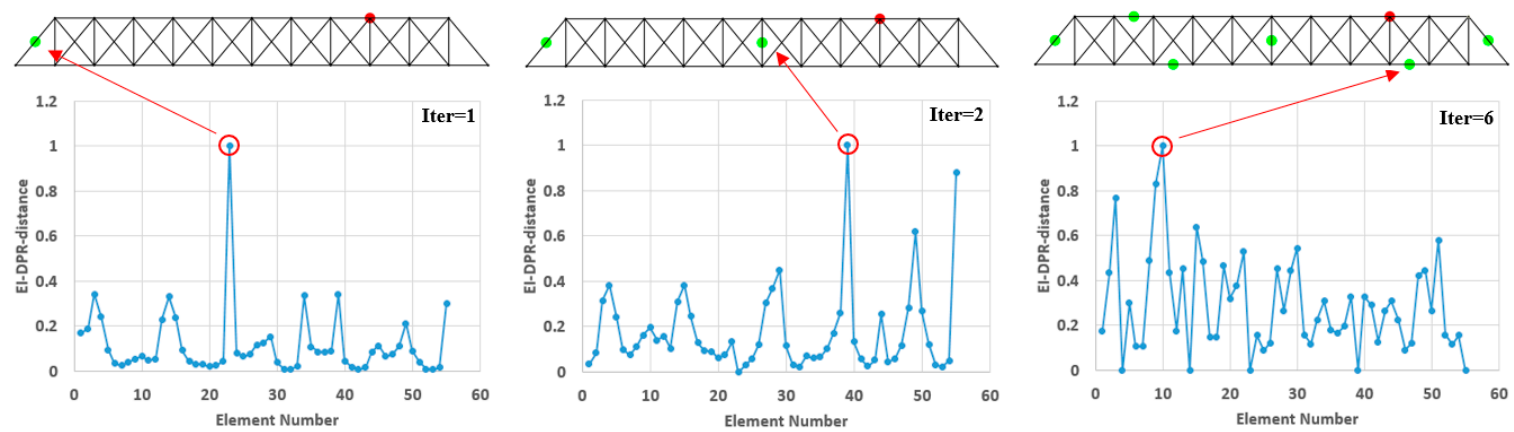

(c)

Figure 15. Procedure example of each sensor placement method (Case 2: strain with one displacement): (a) EI method, (b) EI-DPR method, (c) EI-DPR-distance method.

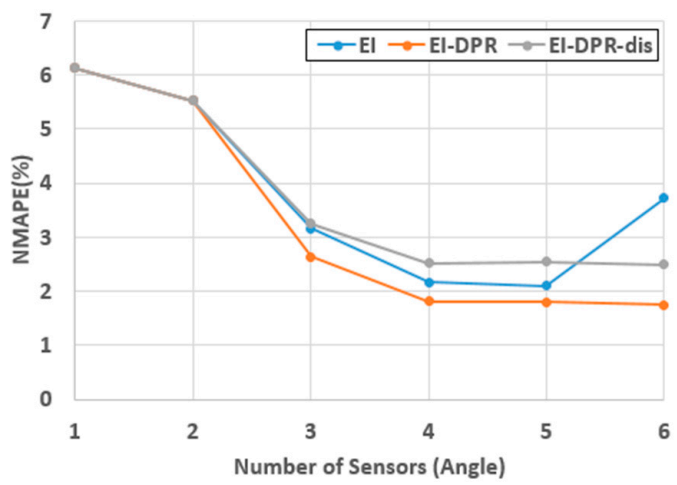

(a)

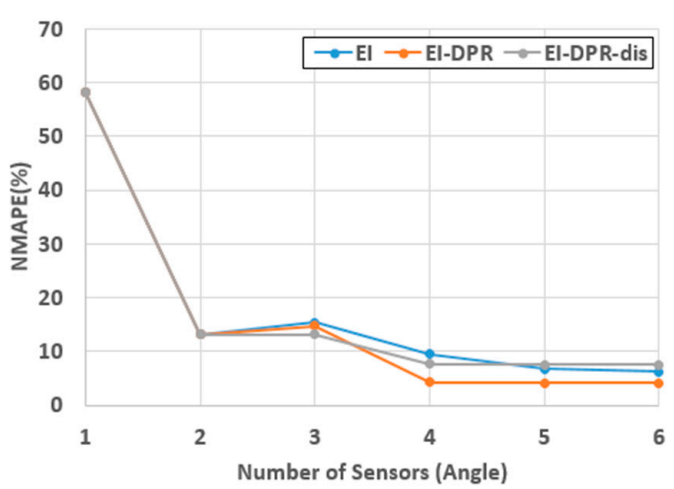

(b)

Figure 16. Cont. 


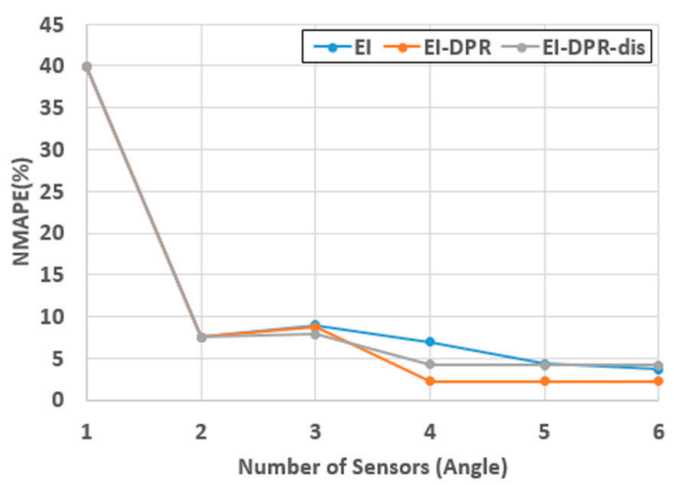

(c)

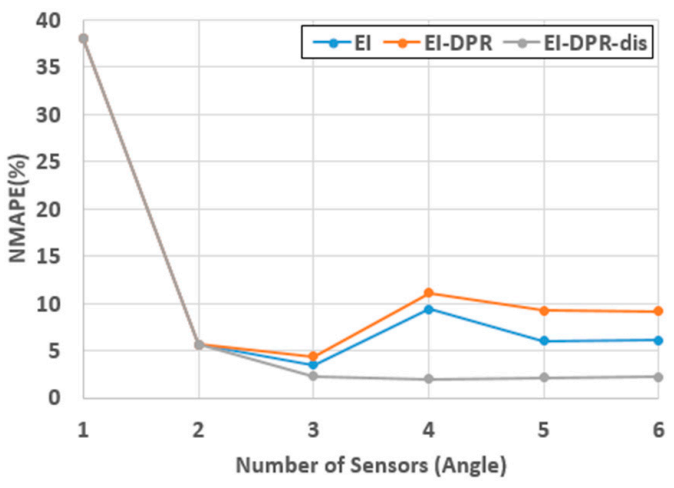

(d)

Figure 16. NMAPE by each sensor placement method according to the number of sensors (Case 1 : slope only): (a) RDS1, (b) RDS2, (c) RDS3, (d) RDS4.

As a result of reviewing the EDS of the case that included the error increase phenomenon, it was found that this occurred as the error of the estimated shape at a location far from the sensors increased, owing to the interference of sensors at a nearby location. The EI-DPR-distance method can minimize the interference effect by considering the distance between the sensors. In addition, according to the required number of sensors to estimate the deformed shape within a 5\% error in Figure 18, it can be seen that the EI-DPR-distance method showed generally better performance than other sensor placement methods. Therefore, the EI-DPR-distance method was adopted as a sensor placement method to verify the effectiveness of using multi-response data.

\subsection{Effectiveness of Using Multi-Response Data}

In this study, the shape function type and sensor placement method were analyzed before validating the use of multi-response data. As a result of the comparison of the shape function type, the SSF was found to be more useful than the MSF for the truss model, which has a relatively complex geometric shape. In addition, the EI-DPR-distance method is proposed using the SSF, where the number of shape functions is larger than the number of sensors. The EI-DPR-distance method can minimize the interference effect between different types of sensors by using the distance coefficient. As a result of the comparison of the sensor placement method, the estimation results corresponding to EI-DPR-distance were generally better than those obtained by the EI and EI-DPR methods.

Therefore, the effectiveness of using multi-response data for RDS1-4 was verified using the SSF and EI-DPR-distance methods. For the combination of response data, three cases were considered: displacement with slope, displacement with strain, and displacement with slope and strain. The estimation results obtained using multi-response were compared to those obtained using only displacement to verify the effectiveness. Using only displacement data is the estimation method proposed by Choi et al. [20]. In addition, 
the total number of considered data points for each response was six. The position of the displacement sensors is first determined by considering the position of the boundary condition, and the slope sensors are placed according to the position of the displacement sensors. The strain sensors are finally placed considering the position of the displacement and slope sensors.

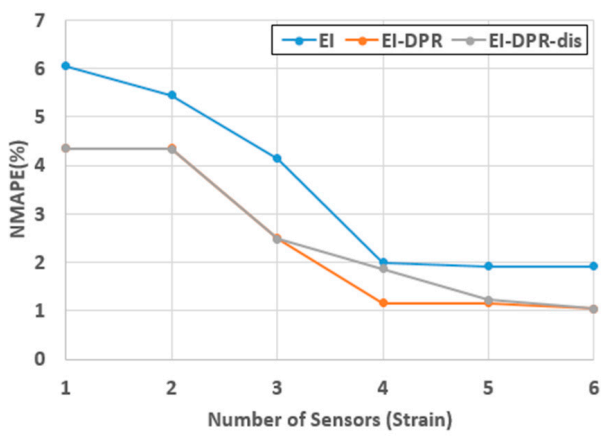

(a)

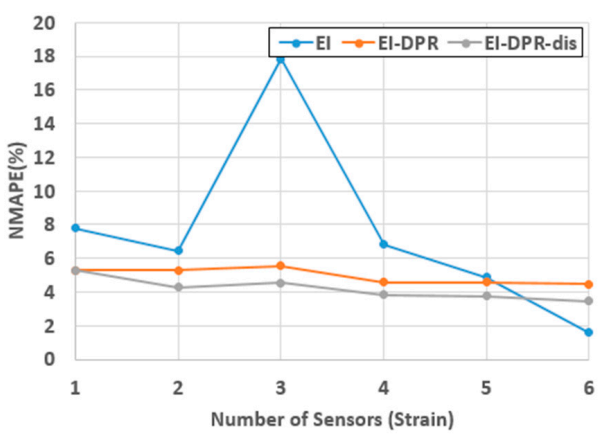

(b)

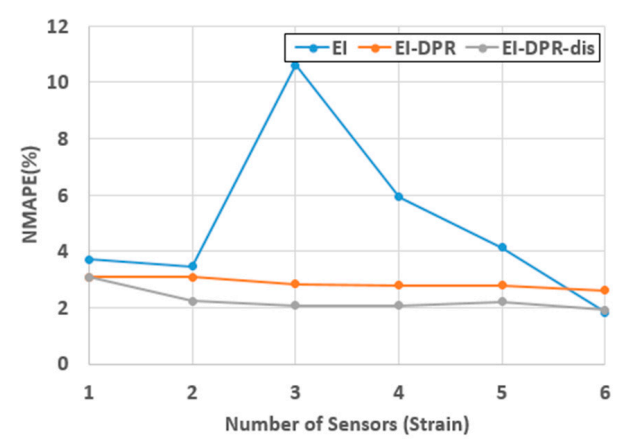

(c)

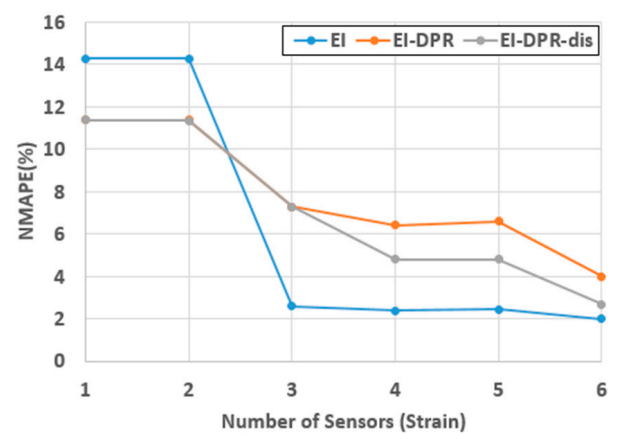

(d)

Figure 17. NMAPE by each sensor placement method according to the number of sensors (Case 2: strain with one displacement): (a) RDS1, (b) RDS2, (c) RDS3, (d) RDS4. 


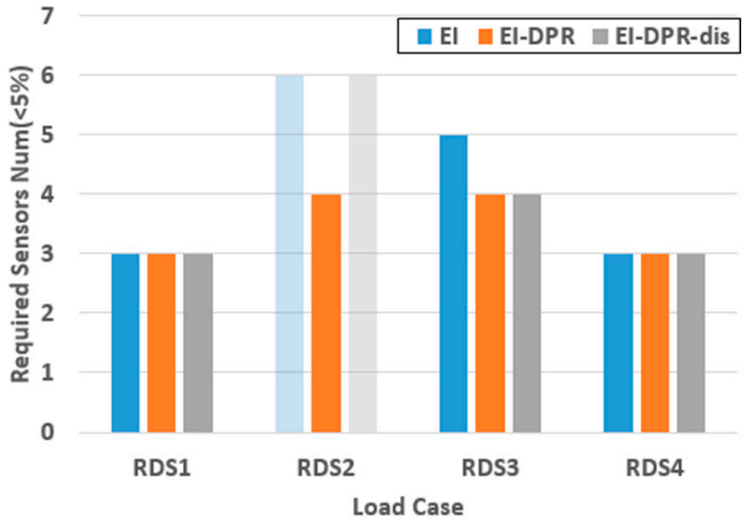

(a)

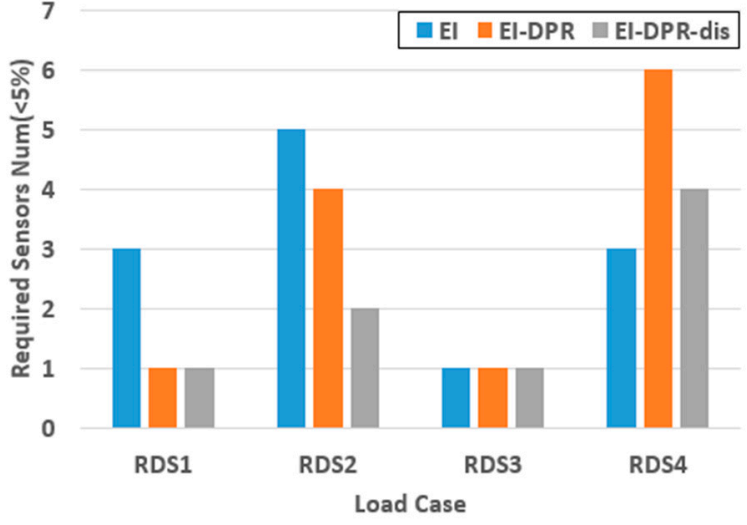

(b)

Figure 18. Required number of sensors by each sensor placement method (NMAPE < 5\%): (a) Case 1: slope only; (b) Case 2: strain with one displacement.

\subsubsection{Beam Model}

The multi-response sensors are placed in the order of displacement, slope, and strain using the EI-DPR-distance method. Due to the fact that there are too many combinations of sensor locations, the sensor locations for the case of using only two-response sensors are shown in Tables 6 and 7. For the beam mode, the slope and strain data are derived from the node and element, respectively. Examples of EDSs based on the combinations of sensors are shown in Figure 19. The red, blue, and green circles indicate the displacement, slope, and strain, respectively. As shown in Figure 19, the estimation accuracy using slope and strain data was more accurate than that using only displacement data.

Table 6. Sensor location in the FEM model used for estimation (slope with displacement)—beam.

\begin{tabular}{cccccccc}
\hline \multicolumn{2}{c}{ Displacement (Node) } & \multicolumn{7}{c}{ Number of Slope Data (Node) } \\
\hline Number of Data & {$[20]$} & $\varnothing \mathbf{1}$ & $\varnothing \mathbf{2}$ & $\varnothing \mathbf{3}$ & $\varnothing \mathbf{4}$ & $\varnothing \mathbf{5}$ & $\varnothing \mathbf{6}$ \\
\hline$\omega 1$ & 8 & 61 & 31 & 1 & 1 & 1 & 1 \\
$\omega 2$ & 24 & 31 & 1 & 61 & 61 & 61 & 61 \\
$\omega 3$ & 23 & 46 & 61 & 45 & 31 & 31 & 31 \\
$\omega 4$ & 39 & 1 & 17 & 33 & 16 & 46 & 17 \\
$\omega 5$ & 11 & 17 & 45 & 16 & 46 & 17 & 45 \\
$\omega 6$ & 51 & 13 & 13 & 28 & 4 & 14 & 48 \\
\hline
\end{tabular}

Table 7. Sensor location in the FEM model used for estimation (strain with displacement)—beam.

\begin{tabular}{cccccccc}
\hline \multicolumn{2}{c}{ Displacement (Node) } & \multicolumn{7}{c}{ Number of Strain Data (Element) } \\
\hline Number of Data & {$[20]$} & $\boldsymbol{\varepsilon} \mathbf{1}$ & $\boldsymbol{\varepsilon} \mathbf{2}$ & $\boldsymbol{\varepsilon} \mathbf{3}$ & $\boldsymbol{\varepsilon} \mathbf{4}$ & $\boldsymbol{\varepsilon} 5$ & $\boldsymbol{\varepsilon} \mathbf{6}$ \\
\hline $\boldsymbol{\omega 1}$ & 8 & 54 & 24 & 38 & 30 & 30 & 30 \\
$\omega 2$ & 24 & 37 & 39 & 30 & 15 & 46 & 16 \\
$\omega 3$ & 23 & 22 & 16 & 45 & 46 & 16 & 45 \\
$\omega 4$ & 39 & 45 & 31 & 15 & 57 & 57 & 57 \\
$\omega 5$ & 11 & 30 & 46 & 57 & 4 & 4 & 4 \\
$\omega 6$ & 51 & 15 & 20 & 4 & 11 & 50 & 35 \\
\hline
\end{tabular}

The estimation errors for each RDS according to the combination of response data are shown in Figures 20-23. The maximum estimation errors were 16.99\% for RDS1, 19.89\% for RDS2, $24.95 \%$ for RDS3, and $27.53 \%$ for RDS4. For all RDS, the estimation errors were reduced according to the additional number of slope and strain data. In addition, estimation accuracies using the slope and strain data were more improved than those using the slope and slope data, respectively. Particularly in RDS3, the estimation error was 
reduced by approximately $20 \%$, with only one additional data point because the second sensor was properly placed in a location where the deformed shape could not be captured through the first sensor.

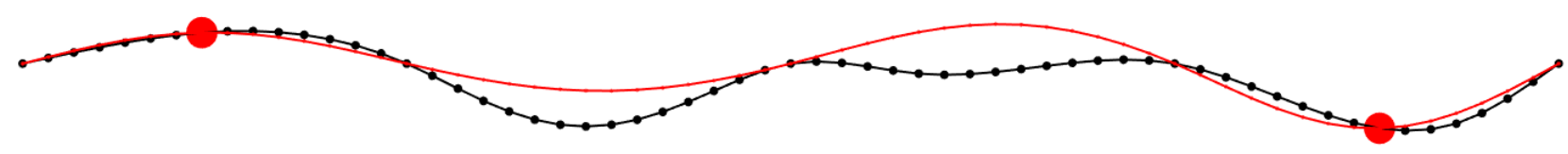

(a)

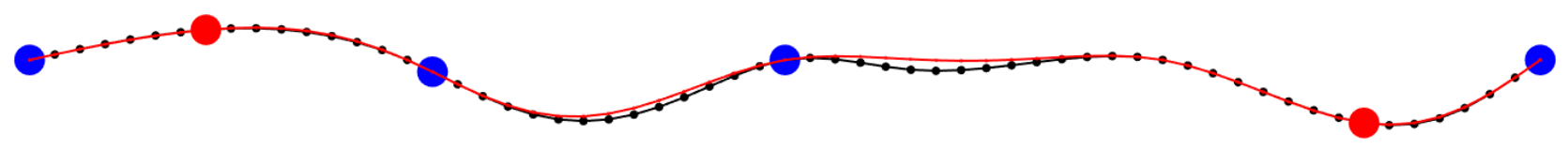

(b)

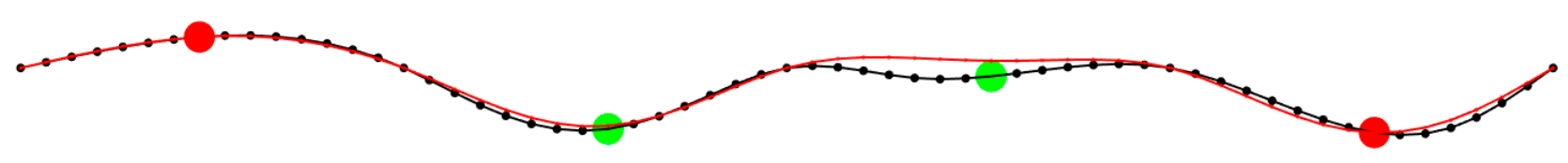

(c)

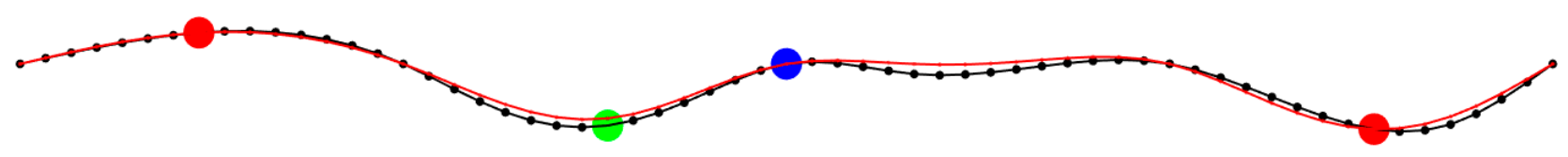

(d)

Figure 19. Example of estimated deformed shape according to the sensor number and combination (RDS4): (a) number of data $(\omega=2) ;(\mathbf{b})$ number of data $(\omega=2, \varnothing=4)$; (c) number of data $(\omega=2, \varepsilon=2)$; (d) number of data $(\omega=2, \varnothing=1, \varepsilon=1)$.

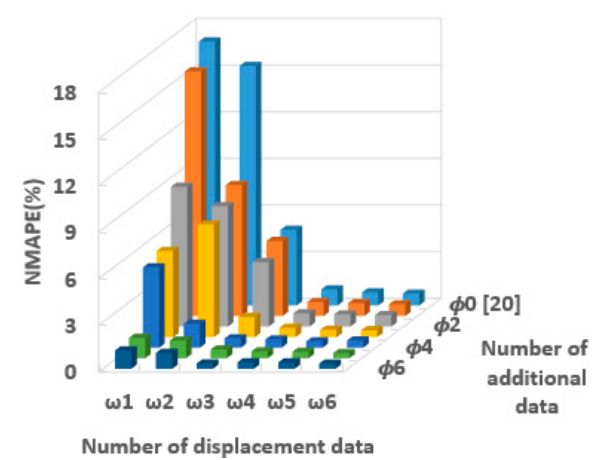

(a)

Figure 20. Cont. 


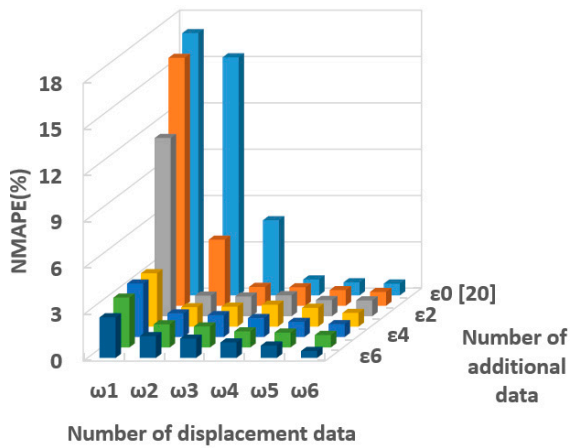

(b)

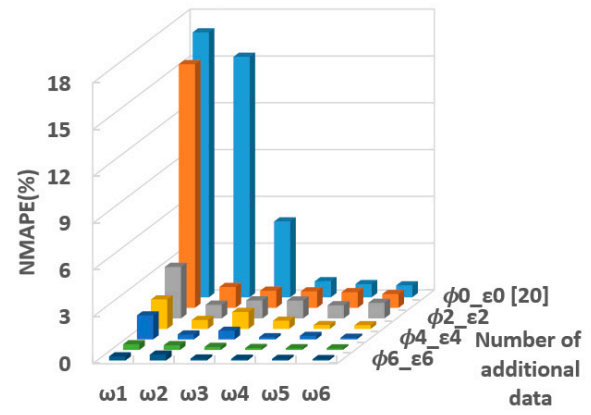

Number of displacement data

(c)

Figure 20. NMAPE according to the number and combination of sensors (RDS1): (a) displacement + slope, (b) displacement + strain, (c) displacement + slope + strain.

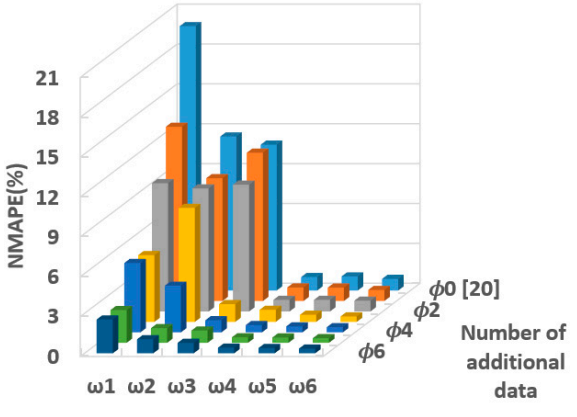

Number of displacement data

(a)

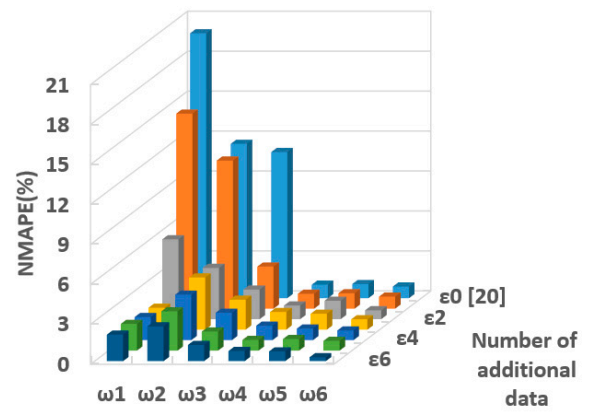

Number of displacement data

(b)

Figure 21. Cont. 


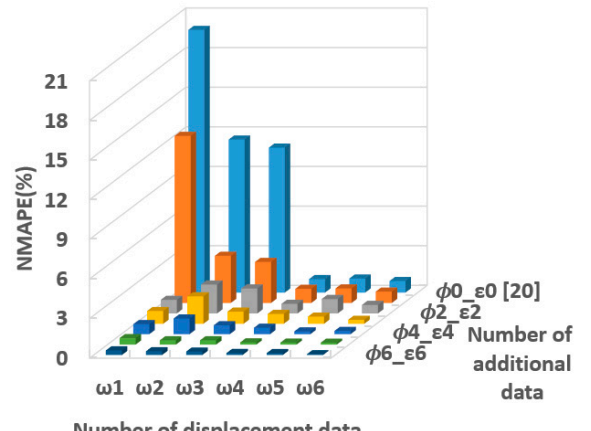

(c)

Figure 21. NMAPE according to the number and combination of sensors (RDS2): (a) displacement + slope, (b) displacement + strain, (c) displacement + slope + strain.

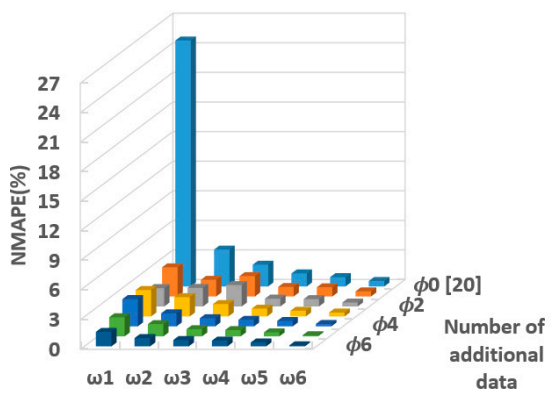

Number of displacement data

(a)

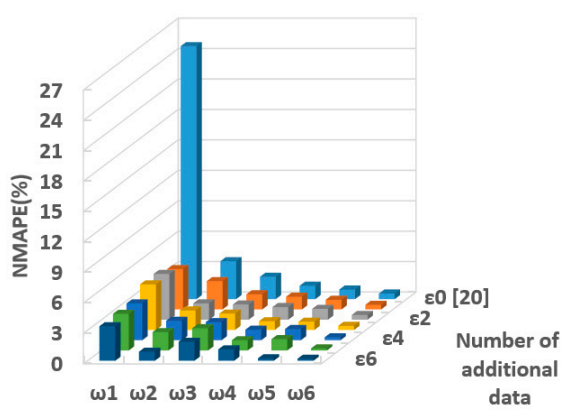

Number of displacement data

(b)

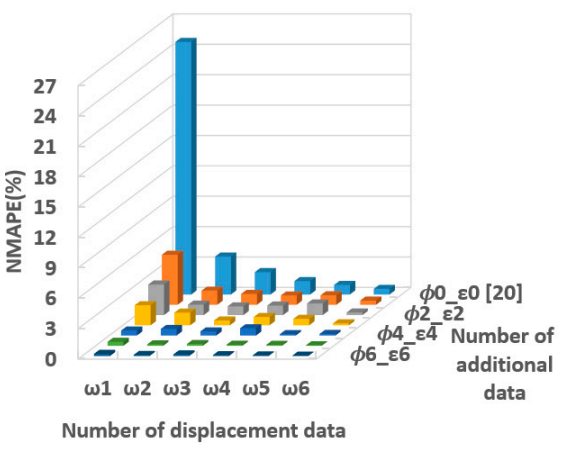

(c)

Figure 22. NMAPE according to the number and combination of sensors (RDS3): (a) displacement + slope, (b) displacement + strain, (c) displacement + slope + strain. 


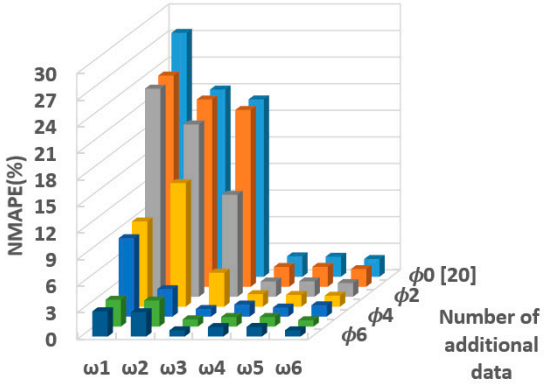

Number of displacement data

(a)

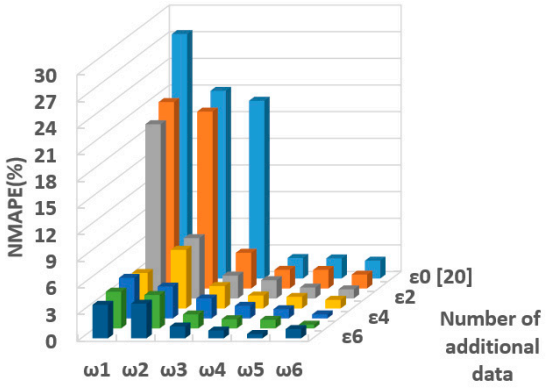

Number of displacement data

(b)

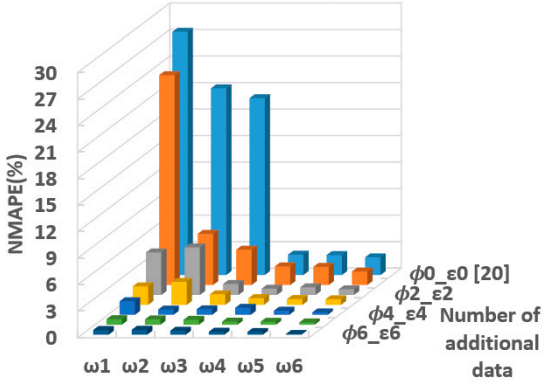

Number of displacement data

(c)

Figure 23. NMAPE according to the number and combination of sensors (RDS4): (a) displacement + slope, (b) displacement + strain, (c) displacement + slope + strain.

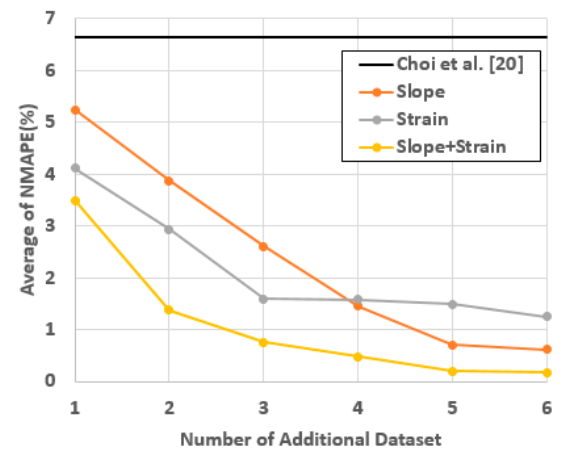

(a)

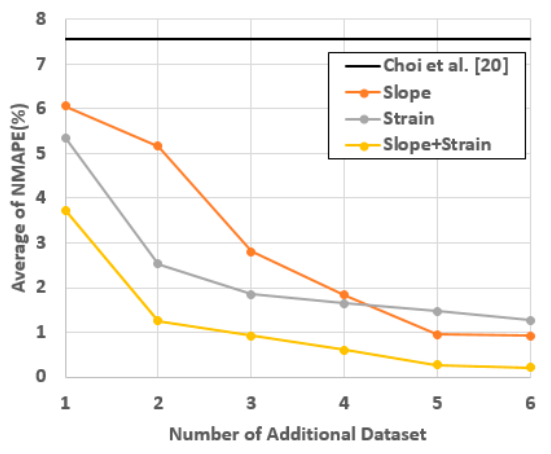

(b)

Figure 24. Cont. 


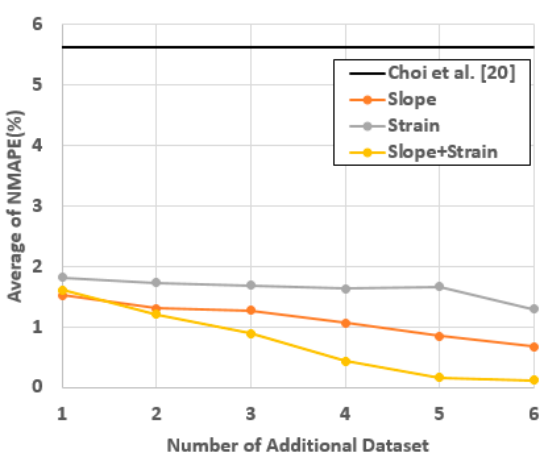

(c)

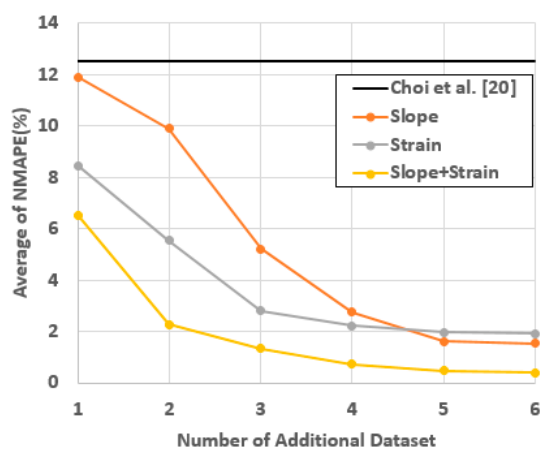

(d)

Figure 24. Mean NMAPE for displacement by additional response data: (a) RDS1, (b) RDS2, (c) RDS3, (d) RDS4.

The average errors for the displacement data number are presented in Figure 22 to evaluate the effect of using multi-response data more clearly. For all RDS, the trend of improving accuracy owing to the additional slope and strain data can be clearly seen in Figure 24. In particular, in the case of using slope and strain data together, a reduced level of estimation error was the highest. Therefore, the algorithm developed in this study can be utilized to efficiently estimate the deformed shape of the beam model by comprehensively using data on the slope, strain, and displacement.

\subsubsection{Truss Model}

In the same way as for the beam model, the locations of displacement, slope, and strain sensors are determined using the EI-DPR-distance method. The sensor arrangements for the two-response case are presented in Tables 8 and 9. For the truss model, both the slope and strain data were derived from the element. Examples of deformed shapes estimated using various combinations of sensors are shown in Figure 25. The red, blue, and green circles indicate the displacement, slope, and strain, respectively. As a result of the estimation, the accuracies of the truss model are improved by using slope and strain data.

The estimation errors for each RDS according to the combination of response data are shown in Figures 26-29. The maximum estimation error was 3.75\% for RDS1, 5.07\% for RDS2, 4.23\% for RDS3, and 14.19\% for RDS4. For all RDS, when slope and strain data were considered in addition to the displacement data, the tendency of the error reduction according to the additional number of data was the same as that of the beam model. However, because the truss model has a lower number of spans than the beam model, the deformed shape that can occur is not diverse. Therefore, the estimation errors for the truss model are generally fewer than those for the beam model.

Table 8. Sensor location in the FEM model used for estimation (slope with displacement) - truss.

\begin{tabular}{cccccccc}
\hline \multicolumn{2}{c}{ Displacement (Node) } & \multicolumn{5}{c}{ Number of Slope Data (Element) } \\
\hline Number of Data & {$[20]$} & $\varnothing \mathbf{1}$ & $\varnothing \mathbf{2}$ & $\varnothing \mathbf{3}$ & $\varnothing \mathbf{4}$ & $\varnothing \mathbf{5}$ & $\varnothing \mathbf{6}$ \\
\hline$\omega 1$ & 22 & 1 & 12 & 6 & 18 & 17 & 7 \\
$\omega 2$ & 16 & 1 & 12 & 7 & 17 & 18 & 6 \\
$\omega 3$ & 11 & 1 & 7 & 12 & 17 & 18 & 6 \\
$\omega 4$ & 3 & 7 & 1 & 12 & 17 & 18 & 6 \\
$\omega 5$ & 5 & 1 & 12 & 18 & 6 & 17 & 7 \\
$\omega 6$ & 9 & 1 & 12 & 17 & 7 & 18 & 6 \\
\hline
\end{tabular}


Table 9. Sensor location in the FEM model used for estimation (strain with displacement)—truss.

\begin{tabular}{cccccccc}
\hline \multicolumn{2}{c}{ Displacement (Node) } & \multicolumn{7}{c}{ Number of Strain Data (Element) } \\
\hline Number of Data & {$[\mathbf{2 0}$} & $\boldsymbol{\varepsilon} \mathbf{1}$ & $\boldsymbol{\varepsilon} \mathbf{2}$ & $\boldsymbol{\varepsilon} \mathbf{3}$ & $\boldsymbol{\varepsilon} \mathbf{4}$ & $\boldsymbol{\varepsilon} \mathbf{5}$ & $\boldsymbol{\varepsilon} \mathbf{6}$ \\
\hline $\boldsymbol{\omega 1}$ & 22 & 23 & 39 & 55 & 4 & 14 & 10 \\
$\boldsymbol{\omega} 2$ & 16 & 39 & 23 & 55 & 3 & 10 & 9 \\
$\omega 3$ & 11 & 39 & 23 & 55 & 3 & 9 & 4 \\
$\omega 4$ & 3 & 39 & 23 & 55 & 9 & 4 & 13 \\
$\omega 5$ & 5 & 39 & 23 & 55 & 9 & 13 & 22 \\
$\omega 6$ & 9 & 39 & 23 & 55 & 13 & 22 & 49 \\
\hline
\end{tabular}

For RDS1-3, where the deformed shape could be estimated with an error of less than approximately $5 \%$ using only one displacement data, the effect of reducing the error by adding slope and strain data did not appear to be significant. By contrast, for RDS4 with a large maximum error, additional slope and strain data significantly affected the improvement of the estimation accuracy. That is, when a sufficient number of displacement sensors to satisfy the target error are already installed, the effect of the addition of the slope and strain sensors may be small.

The average errors for the displacement data number are presented in Figure 30 to more clearly evaluate the effect of using multi-response data. As with the beam model, the estimation error tended to be reduced as the additional number of slope and strain data increased for all RDS. In particular, the deformed shape could be more effectively estimated by adding the slope and strain data together. Therefore, the developed algorithm using multi-response data is also effective for estimating the deformed shape of the truss model.

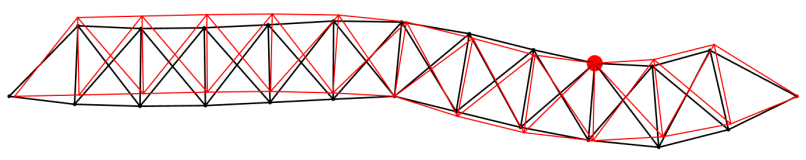

(a)

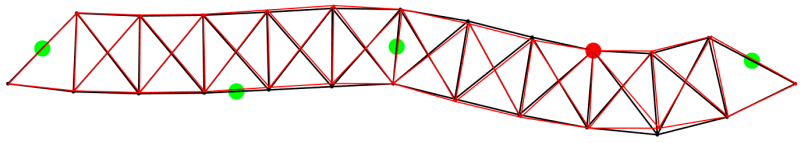

(c)

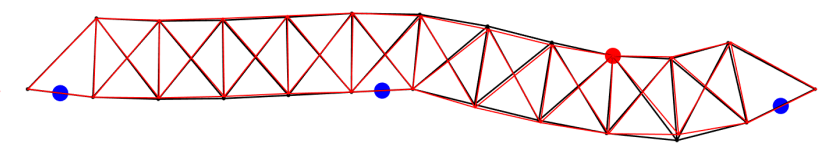

(b)

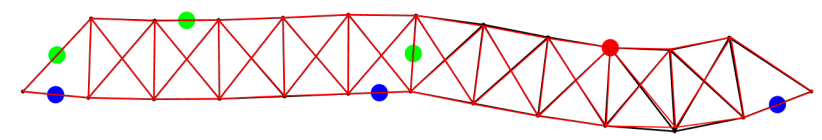

(d)

Figure 25. Example of estimated deformed shape according to the sensor number and combination (RDS4): (a) number of data $(\omega=1),(\mathbf{b})$ number of data $(\omega=1, \varnothing=3)$, (c) number of data $(\omega=1, \varepsilon=4),(\mathbf{d})$ number of data $(\omega=1, \varnothing=3, \varepsilon=3)$.

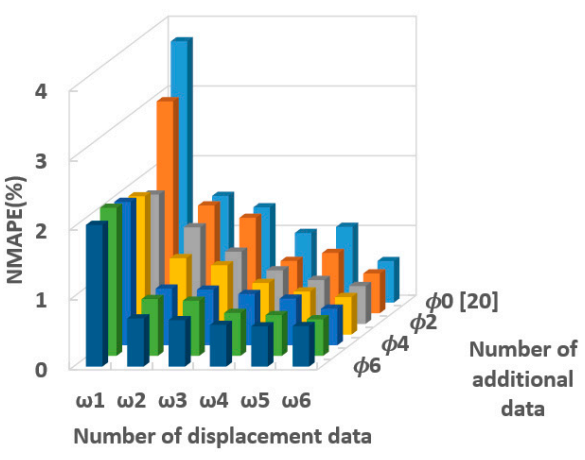

(a)

Figure 26. Cont. 


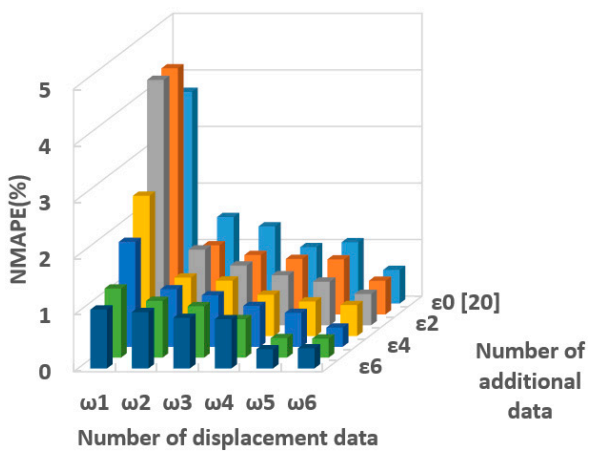

(b)

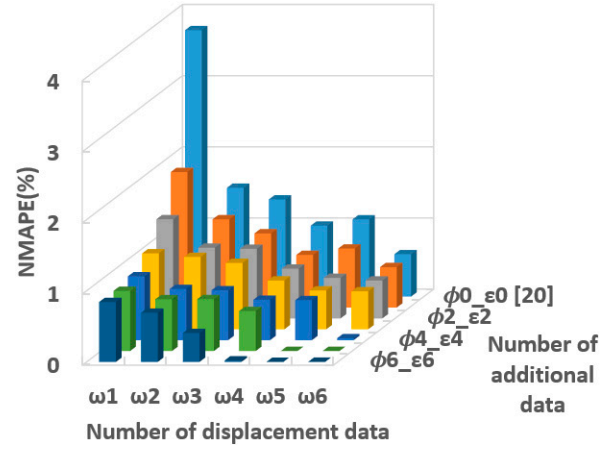

(c)

Figure 26. NMAPE according to the number and combination of sensors (RDS1): (a) displacement + slope, (b) displacement + strain, (c) displacement + slope + strain.

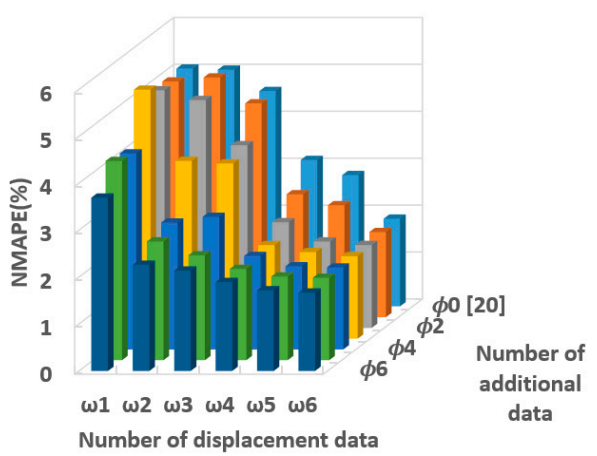

(a)

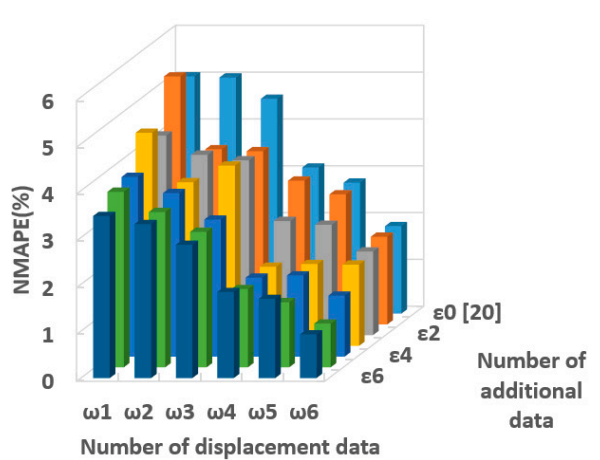

(b)

Figure 27. Cont. 


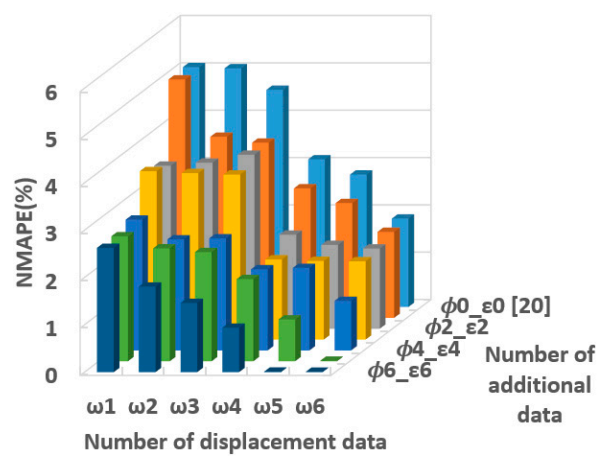

(c)

Figure 27. NMAPE according to the number and combination of sensors (RDS2): (a) displacement + slope, (b) displacement + strain, (c) displacement + slope + strain.

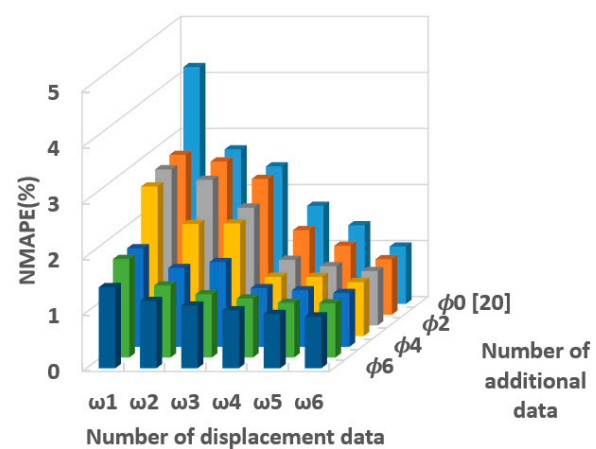

(a)

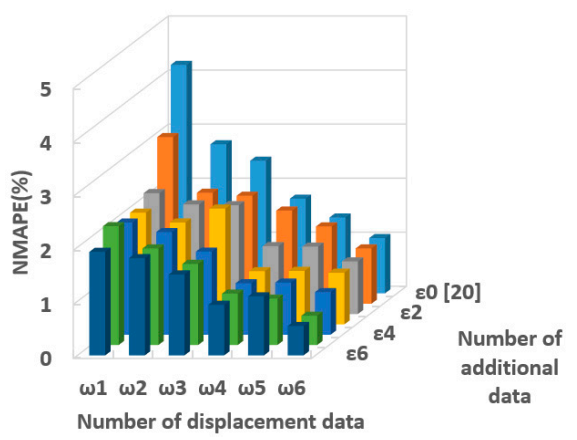

(b)

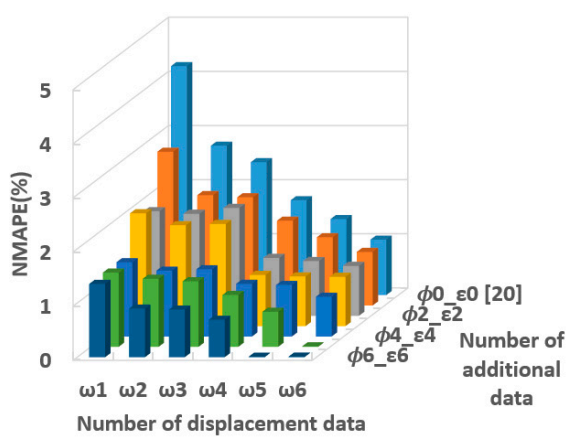

(c)

Figure 28. NMAPE according to the number and combination of sensors (RDS3): (a) displacement + slope; (b) displacement + strain; (c) displacement + slope + strain. 


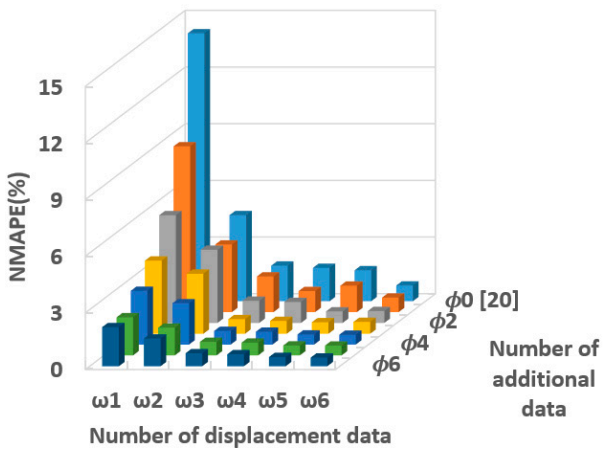

(a)

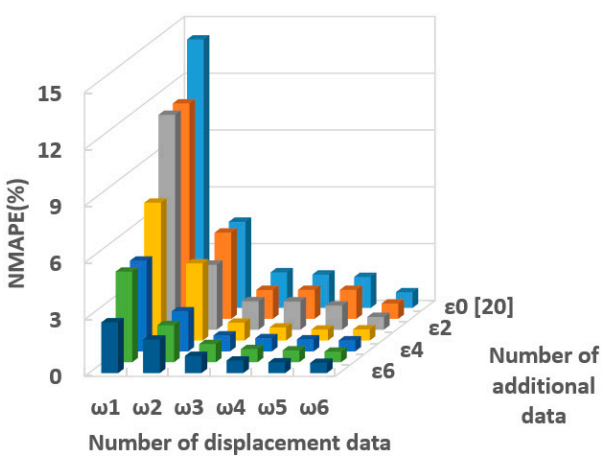

(b)

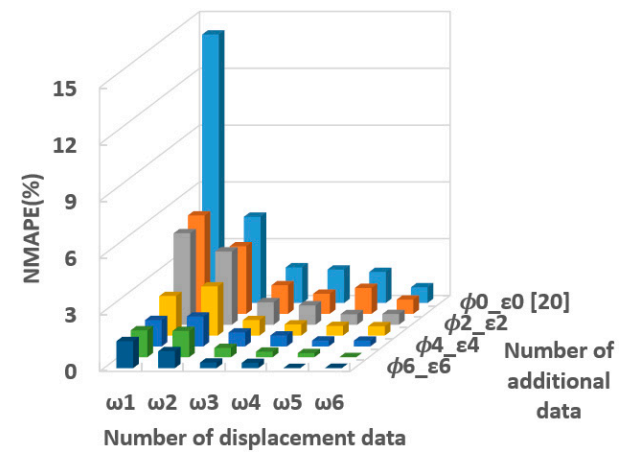

(c)

Figure 29. NMAPE according to the number and combination of sensors (RDS4): (a) displacement + slope, (b) displacement + strain, (c) displacement + slope + strain.

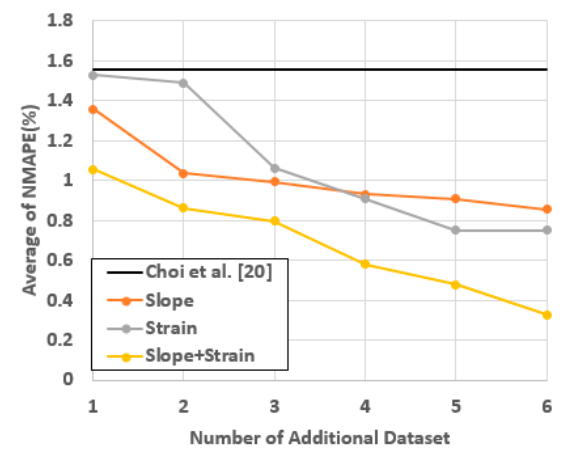

(a)

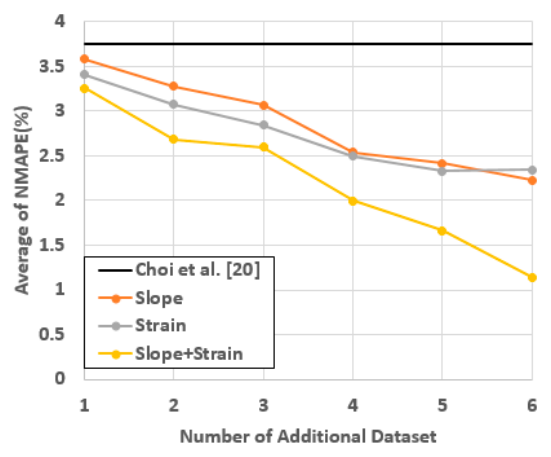

(b)

Figure 30. Cont. 


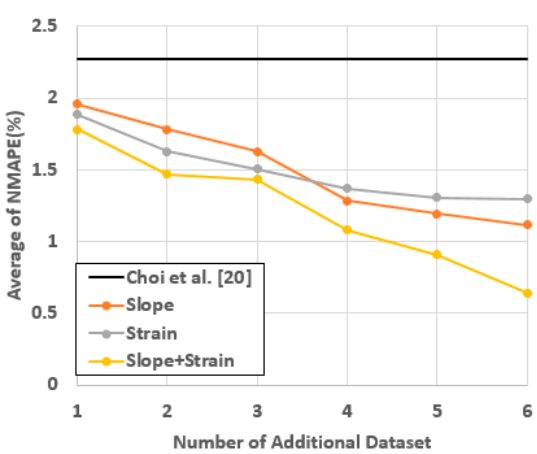

(c)

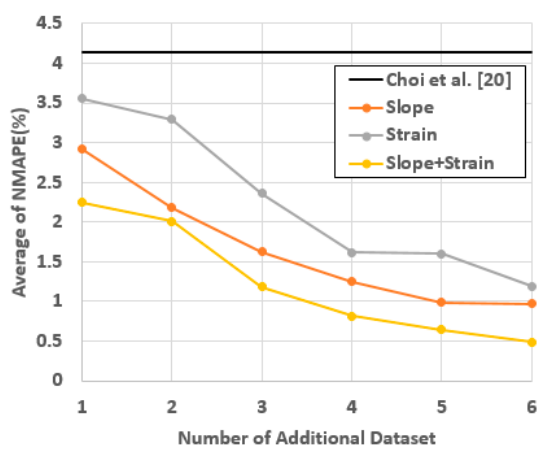

(d)

Figure 30. Mean NMAPE for displacement by additional response data: (a) RDS1, (b) RDS2, (c) RDS3, (d) RDS4.

\section{Conclusions}

In this study, an algorithm that can estimate SDS using displacement data combined with an additional slope and strain data was proposed, and its effectiveness was verified using an FEM model. In the validation process, three issues that can affect the estimation results were analyzed. The main findings derived from the validation process are as follows.

- Comparison of results by MSF and SSF has shown that SSF is more useful for estimating structural deformed shape. In addition, EI-DPR-distance, the sensor placement method proposed in this study, can minimize the interference effect between adjacent sensors and estimate the deformed shape with more stable accuracy than the EI and EI-DPR methods.

- Finally, an estimation algorithm using multi-response data has shown better performance compared with previous work. The addition of slope and strain data can improve estimation accuracy or reduce required displacement data to estimate rational SDS. Therefore, it is expected that cost-effective SHM can be established using the proposed estimation method.

- However, the target model verified in this study is limited to the 2D FEM model. Verification with a 3D FEM model, including transverse response and field tests, is required to be performed as a further study in order to apply the developed algorithm to the SHM of an actual structure.

Author Contributions: Supervision, Y.-J.K.; Writing original draft, N.B.; Writing review \& editing, J.L. and K.L. All authors have read and agreed to the published version of the manuscript.

Funding: This work was supported by a National Research Foundation of Korea (NRF) grant funded by the Korean Government (MIST) (No. 2020R1A2C201445012).

Institutional Review Board Statement: Not applicable.

Informed Consent Statement: Not applicable.

Data Availability Statement: The data presented in this study are available on request from the corresponding author.

Conflicts of Interest: The authors declare no conflict of interest.

\section{References}

1. Chae, M.; Yoo, H.; Kim, J.; Cho, M. Development of a wireless sensor network system for suspension bridge health monitoring. Autom. Constr. 2012, 21, 237-252. [CrossRef]

2. Zhou, G.-D.; Yi, T.-H. Recent Developments on Wireless Sensor Networks Technology for Bridge Health Monitoring. Math. Probl. Eng. 2013, 2013, 1-33. [CrossRef]

3. Chen, B.; Wang, X.; Sun, D.; Xie, X. Integrated System of Structural Health Monitoring and Intelligent Management for a Cable-Stayed Bridge. Sci. World J. 2014, 2014, 1-12. [CrossRef] [PubMed] 
4. Marchewka, A.; Ziółkowski, P.; Aguilar-Vidal, V. Framework for Structural Health Monitoring of Steel Bridges by Computer Vision. Sensors 2020, 20, 700. [CrossRef] [PubMed]

5. Rashidi, M.; Mohammadi, M.; Kivi, S.S.; Abdolvand, M.; Truong-Hong, L.; Samali, B. A Decade of Modern Bridge Monitoring Using Terrestrial Laser Scanning: Review and Future Directions. Remote Sens. 2020, 12, 3796. [CrossRef]

6. Park, K.-T.; Kim, S.-H.; Park, H.-S.; Lee, K.-W. The determination of bridge displacement using measured acceleration. Eng. Struct. 2005, 27, 371-378. [CrossRef]

7. Lee, H.S.; Hong, Y.H.; Park, H.W. Design of an FIR filter for the displacement reconstruction using measured acceleration in low-frequency dominant structures. Int. J. Numer. Methods Eng. 2009, 82, 403-434. [CrossRef]

8. Park, J.-W.; Sim, S.-H.; Jung, H.-J.; Spencer, B.F., Jr. Development of a Wireless Displacement Measurement System Using Acceleration Responses. Sensors 2013, 13, 8377-8392. [CrossRef]

9. Cho, S.; Sim, S.-H.; Park, J.-W.; Lee, J. Extension of indirect displacement estimation method using acceleration and strain to various types of beam structures. Smart Struct. Syst. 2014, 14, 699-718. [CrossRef]

10. Cho, S.; Yun, C.-B.; Sim, S.-H. Displacement estimation of bridge structures using data fusion of acceleration and strain measurement incorporating finite element model. Smart Struct. Syst. 2015, 15, 645-663. [CrossRef]

11. Hou, X.; Yang, X.; Huang, Q. Using Inclinometers to Measure Bridge Deflection. J. Bridg. Eng. 2005, 10, 564-569. [CrossRef]

12. Foss, G.C.; Haugse, E.D. Using modal test results to develop strain to displacement transformation. In Proceedings of the 13th International Modal Analysis Conference, Nashville, TN, USA, 13-16 February 1995; pp. 112-118.

13. Shin, S.; Lee, S.-U.; Kim, Y.; Kim, N.-S. Estimation of bridge displacement responses using FBG sensors and theoretical mode shapes. Struct. Eng. Mech. 2012, 42, 229-245. [CrossRef]

14. Kang, L.-H.; Kim, D.-K.; Han, J.-H. Estimation of dynamic structural displacements using fiber Bragg grating strain sensors. J. Sound Vib. 2007, 305, 534-542. [CrossRef]

15. Rapp, S.; Kang, L.-H.; Han, J.-H.; Mueller, U.C.; Baier, H. Displacement field estimation for a two-dimensional structure using fiber Bragg grating sensors. Smart Mater. Struct. 2009, 18, 025006. [CrossRef]

16. Cho, S.; Park, J.-W.; Palanisamy, R.P.; Sim, S.-H. Reference-Free Displacement Estimation of Bridges Using Kalman Filter-Based Multimetric Data Fusion. J. Sens. 2016, 2016, 1-9. [CrossRef]

17. Li, L.; Zhong, B.-S.; Li, W.-Q.; Sun, W.; Zhu, X.-J. Structural shape reconstruction of fiber Bragg grating flexible plate based on strain modes using finite element method. J. Intell. Mater. Syst. Struct. 2017, 29, 463-478. [CrossRef]

18. Deng, H.; Zhang, H.; Wang, J.; Zhang, J.; Ma, M.; Zhong, X. Modal learning displacement-strain transformation. Rev. Sci. Instrum. 2019, 90, 075113. [CrossRef] [PubMed]

19. Kliewer, K.; Glisic, B. A Comparison of Strain-Based Methods for the Evaluation of the Relative Displacement of Beam-Like Structures. Front. Built Environ. 2019, 5, 118. [CrossRef]

20. Choi, J.; Lee, K.; Kang, Y. Evaluation of quasi-static responses using displacement data from a limited number of points on a structure. Int. J. Steel Struct. 2017, 17, 1211-1224. [CrossRef]

21. Choi, J.; Lee, K.; Kang, Y. Quasi-static responses estimation of a cable-stayed bridge from displacement data at a limited number of points. Int. J. Steel Struct. 2017, 17, 789-800. [CrossRef]

22. Datta, B.N. Numerical Linear Algebra and Applications, 2nd ed.; John Wiley \&Sons: Hoboken, NJ, USA, 2010.

23. Meo, M.; Zumpano, G. On the optimal sensor placement techniques for a bridge structure. Eng. Struct. 2005, $27,1488-1497$. [CrossRef]

24. Worden, K.; Burrows, A. Optimal sensor placement for fault detection. Eng. Struct. 2001, 23, 885-901. [CrossRef]

25. Sunca, F.; Okur, F.Y.; Altunişik, A.C.; Kahya, V. Optimal Sensor Placement for Laminated Composite and Steel Cantilever Beams by the Effective Independence Method. Struct. Eng. Int. 2021, 31, 85-92. [CrossRef]

26. Kammer, D. Sensor Placement for On-Orbit Modal Identification and Correlation of Large Space Structures. J. Guid. Control Dyn. 1991, 14, 251-259. [CrossRef]

27. Papadopoulos, M.; Garcia, E. Sensor placement methodologies for dynamic testing. AIAA J. 1998, 36, 256-263. [CrossRef] 